\title{
High-resolution simulations of the flow around an impulsively started cylinder using vortex methods
}

\author{
By P. KOUMOUTSAKOS $\dagger$ AND A. LEONARD \\ Graduate Aeronautical Laboratories, California Institute of Technology, Pasadena, \\ CA 91125 , USA
}

(Received 28 March 1994 and in revised form 10 January 1995)

The development of a two-dimensional viscous incompressible flow generated from a circular cylinder impulsively started into rectilinear motion is studied computationally. An adaptative numerical scheme, based on vortex methods, is used to integrate the vorticity/velocity formulation of the Navier-Stokes equations for a wide range of Reynolds numbers $(R e=40$ to 9500$)$. A novel technique is implemented to resolve diffusion effects and enforce the no-slip boundary condition. The Biot-Savart law is employed to compute the velocities, thus eliminating the need for imposing the far-field boundary conditions. An efficient fast summation algorithm was implemented that allows a large number of computational elements, thus producing unprecedented highresolution simulations. Results are compared to those from other theoretical, experimental and computational works and the relation between the unsteady vorticity field and the forces experienced by the body is discussed.

\section{Introduction}

The impulsively started cylinder problem is studied as a prototype of unsteady separated flows and has been the subject of many theoretical, experimental and computational works. Experimental investigations on unsteady flows resulting from an impulsive acceleration of a cylinder date back to the time of Prandtl (1925). However, the most extensive experiments to date seem to be those presented by Bouard \& Coutanceau (1980) for the translating cylinder. They used a specially designed apparatus to produce nearly instantaneous starts and analysed photographically the flow field visualized by solid tracers put in uniform suspension in the fluid. This method produces reasonable results for instantaneous velocity field and streamlines which they use to analyse in detail the topological structure of the flow. However quantities such as the time-dependent force coefficients are not available.

Theoretical investigations of an impulsively started flow were first undertaken by Blasius in 1908 who obtained the first two terms of a time series solution of the boundary layer equations. Subsequently there have been many works attempting to obtain higher-order terms and advance the solution beyond the separation stage, but the most notable are those of Collins \& Dennis $(1973 a, b)$ for analytic and numerical solution processes and Bar-Lev \& Yang (1975). In Collins \& Dennis the problem is formulated in boundary layer variables and an expansion in powers of time is obtained. These expansions are corrected to account for finite Reynolds number $(R e)$ effects and are adjusted to match the uniform flow far from the cylinder. In Bar-Lev \& Yang the vorticity equation is solved by the method of matched asymptotic expansions. Inner (rotational flow) and outer (potential flow) solutions are obtained to third order in time 
and a composite solution is formed. Both works provide extensive information for flow quantities of interest (such as vorticity field, streamlines, body forces) that are valid for short times. The range of validity increases with increasing $R e$. They are the yardstick by which many investigators test their numerical scheme, at least for the initial stage of the flow. More recently Dennis \& Kocabiyik (1991) demonstrated that the complete details of the matching procedure can be established using integral conditions, thus relating the solution procedures of Collins \& Dennis and Bar-Lev \& Yang.

Impulsively started flows present a serious challenge for numerical methods. Difficulties exist in the formulation of the boundary and initial conditions of the problem. High-resolution simulations are necessary for high Reynolds numbers to adequately resolve the singular character of the flow at early times and, as we shall see, to resolve the details of the separation process. The first computations were presented in the late $1950 \mathrm{~s}$ by Payne (1958) for relatively low $R e(40-100)$. Numerous computations have been performed in the last 30 years on this flow but there are still open questions as to whether the numerics interfere with the physics of the problem, especially for high-Re flows. The problem is usually formulated in vorticitystreamfunction variables and Eulerian, Lagrangian and hybrid methods have been used for their discretization. Ta Phuoc Loc (1980) uses a fourth-order scheme to resolve Poisson's equation for the streamfunction and a second-order finite difference scheme for the vorticity transport equation. He presented computations for a range of $\operatorname{Re}(550-1000)$ and detailed diagnostics and comparisons with experimental results. This work was extended to higher Re (3000-9500) by Ta Phuoc Loc \& Bouard (1985). Lecointe \& Piquet (1985) have tested several high-order compact finite difference schemes as well and they present accurate computations for $R e$ up to 550 and tentative simulations for higher values of $R e$. Their diagnostics however are limited and not conclusive. A more recent work is that of Wang \& Dalton (1991). They use a predictor-corrector finite difference scheme for the vorticity transport equation and a fast Poisson solver for the streamfunction. They present results for impulsively started and stopped flows for Re of 102 and 550 .

In vortex methods the most notable studies are those of Smith \& Stansby (1988) and the more recent one of Chang \& Chern (1991). Both investigations use the Cloud-inCell method (CIC) (Christiansen 1973) to convect the vortices but use different techniques to account for viscous effects. Smith \& Stansby use the method of random walks whereas Chang \& Chern use a finite difference scheme on the grid used by the CIC technique to resolve the diffusion operator. Both works takes advantage of the stability properties of vortex methods to extend their computations to very high Reynolds numbers (up to $10^{6}$ in Chang \& Chern). However, it appears that the increase in the $R e$ simulated is not followed by an adequate increase in the resolution. Recently Anderson \& Reider (1993), and Wu et al. (1995) conducted simulations of this flow using finite difference schemes. Their simulations demonstrate the computational difficulties of the problem as very large numbers of grid points were necessary, in both works, to advance the solution up to $T=3.0$ for $R e=9500$.

In the present work we perform unprecedented high-resolution direct numerical simulations using a novel adaptive numerical scheme, based on vortex methods. We use up to a million computational elements by efficiently implementing fast summation algorithms and by introducing a novel scheme for the enforcement of the no-slip condition for the vorticity-velocity formation of the Navier-Stokes equations. We compare our results with some representative experimental, theoretical and computational works and provide benchmark quality simulations for the early stages of the flow around an impulsively started cylinder. Moreover, we identify the underlying 
mechanisms of unsteady separation and describe the effect of these phenomena on the drag coefficient.

In $\S 2$ we present the governing equations and the numerical method. In $\S 3$ we present our fractional-step algorithm and describe a novel enforcement of the no-slip boundary condition which is particularly suitable for the computation of impulsively started flows. Our results and discussion of the relation between the vorticity field and the forces experienced by the body are presented in $\S 4$, followed by conclusions.

\section{The two-dimensional Navier-Stokes equations}

Two-dimensional incompressible unsteady flow of a viscous fluid may be determined by the vorticity transport equation as

$$
\frac{\partial \omega}{\partial t}+\boldsymbol{u} \cdot \boldsymbol{\nabla} \omega=\nu \nabla^{2} \omega
$$

where $\boldsymbol{u}(\boldsymbol{x}, t)$ is the velocity, $\boldsymbol{\omega}=\omega \hat{\boldsymbol{e}}_{z}=\boldsymbol{\nabla} \times \boldsymbol{u}$ the vorticity and $\nu$ denotes the kinematic viscosity. For flow around a solid body, translating with velocity $U_{b}(t)$ and rotating around its centre of mass $\left(x_{b}\right)$ with angular velocity $\Omega(t)$, the velocity of the fluid $(\boldsymbol{u})$ is equal to the velocity of the body $\left(U_{s}\right)$ on the surface of the body $\left(\boldsymbol{x}_{s}\right)$ :

$$
u\left(x_{s}\right)=U_{s}
$$

with $U_{s}=U_{b}(t)+\Omega(t) \hat{e}_{z} \times\left(x_{s}-x_{b}\right)$.

At infinity we have

$$
\boldsymbol{u}(\boldsymbol{x}) \rightarrow \boldsymbol{U}_{\infty} \quad \text { as } \quad|\boldsymbol{x}| \rightarrow \infty,
$$

where $U_{\infty}$ is the free-stream velocity. Using the definition of the vorticity and the continuity $(\nabla \cdot u=0)$ it can be shown that $u$ is related to $\omega$ by the following Poisson equation:

$$
\nabla^{2} \boldsymbol{u}=-\nabla \times \omega .
$$

The velocity-vorticity formulation helps in eliminating the pressure from the unknowns of the equations. However, for bounded domains it introduces additional constraints in the kinematics of the flow field and requires the transformation of the velocity boundary conditions to vorticity form.

\subsection{Particle (vortex) methods}

The present numerical method is based on the discretization of the above equations in a Lagrangian frame using particle (vortex) methods. The vorticity equation (1) may be expressed in a Lagrangian formulation by solving for the vorticity-carrying fluid elements $\left(\boldsymbol{x}_{a}\right)$ based on the following set of equations:

$$
\frac{\mathrm{d} x_{\alpha}}{\mathrm{d} t}=\boldsymbol{u}\left(\boldsymbol{x}_{a}, t\right), \quad \frac{\mathrm{d} \omega}{\mathrm{d} t}=\nu \nabla^{2} \omega .
$$

In the context of particle methods it is desirable to replace the right-hand sides of (3) by integral operators. These operators are discretized using the locations of the particles as quadrature points so that ultimately (3) is replaced by a set of ODEs whose solution is equivalent to the solution of the original set of equations. To this effect the velocity field may be determined by the vorticity field using the Green's function formulation for the solution of Poisson's equation (2):

$$
u=-\frac{1}{2 \pi} \int K(x-y) \times \omega \mathrm{d} y+U_{0}(x, t)
$$


where $U_{0}(x, t)$ contains the contribution from the solid-body rotation and $U_{\infty}$, and $K(z)=z /|z|^{2}$. The use of the Biot-Savart law to compute the velocity field guarantees the enforcement of the boundary condition at infinity.

The Laplacian operator may be approximated by an integral operator (Degond \& Mas-Gallic 1989) as well so that in an infinite domain

$$
\nabla^{2} \omega \approx \int G_{\epsilon}(|x-y|)[\omega(x)-\omega(y)] \mathrm{d} \boldsymbol{y} .
$$

The error of this approximation is of $O\left(\epsilon^{r}+(h / \epsilon)^{m}\right)$ where $r, m$ depend on the choice of the smoothing function $G_{\epsilon}$. In this paper we chose $G_{\epsilon}(z)=\left(2 / \pi \epsilon^{2}\right) \exp \left(-|z|^{2} / 2 \epsilon^{2}\right)$, for which $r=2$ and $m=\infty$. When $\epsilon^{2}=2 \nu \delta t, G_{\epsilon}$ is equivalent to the heat kernel. The additional computational cost associated with the calculation of the exponential is overcome by the use of tabulated values of the function.

The boundary condition $(1 b)$ is enforced by formulating the physical mechanism it describes. The solid wall is the source of vorticity that enters the flow. A vorticity flux $(\partial \omega / \partial n)$ may be determined on the boundary in a way that ensures $(1 b)$ is satisfied. Here a fractional step algorithm is presented that allows the calculation of this vorticity flux (see Koumoutsakos, Leonard \& Pepin 1994). It is shown then that this mechanism of vorticity generation can be expressed by an integral operator as well:

$$
\left.\frac{\mathrm{d} \omega}{\mathrm{d} t}\right|_{b o d y}=\int H(x, y) \frac{\partial \omega}{\partial n}(y) \mathrm{d} y,
$$

where the kernel $H$ is developed in $\S 3$. Using the above integral representations for the right-hand side of (3) we obtain the following set of equations:

$$
\left.\begin{array}{rl}
\frac{\mathrm{d} x_{a}}{\mathrm{~d} t} & =-\frac{1}{2 \pi} \int K\left(\boldsymbol{x}_{a}-\boldsymbol{y}\right) \times \boldsymbol{\omega} \mathrm{d} y+U_{0}\left(x_{a}, t\right), \\
\frac{\mathrm{d} \omega}{\mathrm{d} t} & \approx \nu \int G_{\epsilon}\left(\left|x_{a}-y\right|\right)\left[\omega\left(x_{a}\right)-\omega(y)\right] \mathrm{d} y+\nu \int H\left(x_{a}, y\right) \frac{\partial \omega}{\partial n}(y) \mathrm{d} y .
\end{array}\right\}
$$

In vortex methods, the vorticity field is considered as a discrete sum of the individual vorticity fields of the particles, having core radius $\epsilon$, strength $\Gamma(t)$ and an individual distribution of vorticity determined by the function $\eta_{\epsilon}$, so that

$$
\omega(\boldsymbol{x}, t)=\sum_{n=1}^{N} \Gamma_{n}(t) \eta_{\epsilon}\left(\boldsymbol{x}-\boldsymbol{x}_{n}(t)\right) .
$$

When this expression for the vorticity is substituted in (7) the singular integral operators $K, G$ are convolved with the smooth function $\eta_{\varepsilon}$ and are replaced by smooth operators $K_{\epsilon}, G_{\epsilon}$. These integrals are subsequently discretized using a quadrature having as quadrature points the locations of the particles. Assuming that each particle occupies a region of area $h^{2}$ and that the shape of the body is discretized by $M$ panels then algorithmically the method may be expressed as

$$
\left.\begin{array}{rl}
\frac{\mathrm{d} x_{i}}{\mathrm{~d} t} & =-\frac{1}{2 \pi} \sum_{j=1}^{N} \Gamma_{j} K_{\epsilon}\left(x_{i}-x_{j}\right)+U_{0}\left(x_{i}, t\right), \\
\frac{\mathrm{d} \Gamma_{i}}{\mathrm{~d} t} & =\nu \sum_{j=1}^{N}\left[\Gamma_{j}-\Gamma_{i}\right] G_{\epsilon}\left(\left|x_{i}-\boldsymbol{x}_{j}\right|\right)+\nu \sum_{m=1}^{M} H\left(\boldsymbol{x}_{i}, \boldsymbol{x}_{m}\right) \frac{\partial \omega}{\partial n}\left(\boldsymbol{x}_{m}\right), \\
\Gamma_{i}(0) & =\omega\left(\boldsymbol{x}_{i}, 0\right) h^{2}, \quad i=1,2, \ldots, N .
\end{array}\right\}
$$


The characteristic of the present method is the replacement of the differential operators by integral operators. The advantage in employing integral operators is based on their stability and efficiency. Integral operators are bounded and smoothing, so that discrete approximations have bounded condition number as the mesh is refined. The Lagrangian representation of the convective terms avoids many difficulties associated with its discretization on an Eulerian mesh, such as excess numerical diffusion. However, the accuracy of the method relies on the accuracy of the quadrature rule as information needs to be gathered from the possibly scattered particle positions.

\subsection{Fast vortex methods}

The straightforward method of computing the right-hand side of (8), using (9) for every particle requires $O\left(N^{2}\right)$ operations for $N$ vortex elements. This precludes highresolution studies of bluff body flows with more than say 50000 elements. Recently fast methods have been developed that have operation counts of $O(N \log N)$ (Barnes \& Hut 1986) or $O(N)$ (Greengard \& Rohklin 1987) depending on the details of the algorithm. The basic idea of these methods is to decompose the element population spatially into clusters of particles and build a hierarchy of clusters or a 'tree' - smaller neighbouring clusters combine to form a cluster of the next size up in the hierarchy and so on.

The contribution of a cluster of particles to the velocity of a given vortex can then be computed to desired accuracy if the particle is sufficiently far from the cluster in proportion to the size of the cluster and a sufficiently large number of terms in the multipole expansion is taken. This is the essence of the 'particle-box' method, requiring $O(N \log N)$ operations. One then tries to minimize the work required by maximizing the size of the cluster used while keeping the number of terms in the expansion within a reasonable limit and maintaining a certain degree of accuracy.

The 'box-box' scheme goes one step further as it accounts for box-box interactions as well. These interactions are in the form of shifting the expansions of a certain cluster with the desired accuracy to the centre of another cluster. Then those expansions are used to determine the velocities of the particles in the second cluster. This has as the effect of minimizing the tree traversals for the individual particles, requiring only $O(N)$ operations.

In the present scheme the efficient vectorization of the $O(N)$ scheme allows computations with one CPU min per velocity evaluation for one million vortices on a single processor of a CRAY YMP.

\subsection{Lagrangian grid distortion - remeshing}

The convergence properties of vortex methods with a finite core dictate that the particles must overlap (i.e. $h<\epsilon$ ) at all times (Beale 1986). However, the local strain field of the flow may distort the particle locations thus producing particle clustering in one direction accompanied by an expansion in another direction, similar to that which would occur around a hyperbolic stagnation point in a steady flow. When such a situation occurs the particle locations have to be re-initialized (remeshed) onto a uniform grid while interpolating the old vorticity on the new particle locations.

In our algorithm we use a nine-point scheme to perform this remeshing, conserving the circulation as well as the linear and angular momentum of the vorticity field. More specifically the $i$ th 'old' vortex with circulation $\Gamma_{i}$ and location $\left(x_{i}, y_{i}\right)$ contributes $\Delta \Gamma_{j, i}$ circulation to new mesh point $\left(\tilde{x}_{j}, \tilde{y}_{j}\right)$ according to

$$
\Delta \Gamma_{j, i}=\Gamma_{i} \Lambda\left(\frac{\tilde{x}_{j}-x_{i}}{h}\right) \Lambda\left(\frac{\tilde{y}_{j}-y_{i}}{h}\right),
$$


where the interpolation kernel $A$ is given by

$$
A(u)= \begin{cases}1-u^{2}, & 0 \leqslant|u|<\frac{1}{2} ; \\ \frac{1}{2}(1-u)(2-u) ; & \frac{1}{2} \leqslant|u|<\frac{3}{2} ; \\ 0, & \text { otherwise. }\end{cases}
$$

If the old particle is less than a distance $h$ from a wall the interpolation is modified to maintain the same conservation properties. See Koumoutsakos (1993) for further discussion.

\section{A fractional-step algorithm - the no-slip boundary condition}

The no-slip boundary condition accounts for the generation of vorticity on the surface of the body. The surface of the body acts as a source of vorticity (Lighthill 1963 ) and the task is to relate this vorticity flux on the surface of the body to the noslip condition.

The present vortex method is implemented in a time-stepping algorithm that proceeds by generating the particle trajectories and appropriately modifying the particle strengths. In the present formulation equations (9) are not integrated simultaneously in time but instead a fractional-step algorithm is employed. The governing equations are solved via a splitting scheme that accommodates the enforcement of the no-slip boundary condition.

Let us assume that at the $n$th time step (corresponding to time $t-\delta t$ ) the vorticity field has been computed (respecting the no-slip boundary condition) and we seek to advance the solution to the next time step (time $t$ ). The following two-step procedure is implemented (Koumoutsakos et al. 1994).

Step 1. Using as initial conditions $f(x)=\omega^{n}(x, n \delta t)$ we solve

$$
\left.\begin{array}{rl}
\omega_{t}+\boldsymbol{u} \cdot \nabla \omega=\nu \nabla^{2} \omega & \text { in } \mathscr{D} \times[t-\delta t, t], \\
\omega(\boldsymbol{x}, t-\delta t)=f(\boldsymbol{x}) & \text { in } \mathscr{D},
\end{array}\right\}
$$

where $\mathscr{D}$ denotes the flow domain exterior to the body surface $\partial \mathscr{D}$. Particles are advanced via the Biot-Savart law and their strength is modified based on the scheme of 'particle strength exchange'. The kinematic ('no throughflow') boundary condition is enforced at this stage using a vortex sheet on the surface of the body whose strength is determined by requiring that the tangential velocity inside the surface of the body is zero. An improved boundary element formulation is implemented (Koumoutsakos \& Leonard 1993) that allows us to link the kinematic boundary condition with the no-slip boundary condition at the following step.

Algorithmically then Step 1 may be expressed as

$$
\frac{\mathrm{d} \boldsymbol{x}}{\mathrm{d} t}=u^{n}(x, n \delta t), \quad \frac{\mathrm{d} \omega_{1}^{\prime}}{\mathrm{d} t}=\nu \nabla^{2} \omega_{1}^{\prime} .
$$

At the end of Step 1 a vorticity field $\omega_{1}^{\prime}$ has been established in the fluid.

Step 2. The no-slip boundary condition is enforced in this stage by a vorticity (not particle) creation algorithm. The spurious vortex sheet $(\gamma)$ that is observed on the surface of the body at the end of Step 1 is now computed and then may be translated to a vorticity flux:

$$
\gamma \rightarrow \frac{\partial \omega}{\partial n} \quad \text { on } \quad \partial \mathscr{D} .
$$


The computed vorticity flux generates vorticity in the fluid. The vorticity field is augmented by this viscous mechanism as described by the following set of equations:

$$
\left.\begin{array}{ccc}
\frac{\partial \omega_{2}^{\prime}}{\partial t}-\nu \nabla^{2} \omega_{2}^{\prime}=0 & \text { in } \quad \mathscr{D}[t-\delta t, t], \\
\omega_{2}^{\prime}(\boldsymbol{x}, t-\delta t)=0 & \text { in } \quad \mathscr{D} . \\
\nu \frac{\partial \omega_{2}^{\prime}}{\partial n}=\frac{\gamma\left(\omega_{1}^{\prime}\right)}{\delta t} & \text { on } \quad \partial \mathscr{D} .
\end{array}\right\}
$$

Note that the diffusion equation is solved here with homogeneous initial conditions as the initial vorticity field was taken into account in the previous substep. The solution at Step 2 is a vorticity field $\omega_{2}^{\prime}$ which we superimpose onto the solution of Step 1 to obtain the vorticity distribution at the next time step:

$$
\omega^{n+1}=\omega_{1}^{\prime}+\omega_{2}^{\prime} .
$$

\subsection{A note on impulsively started flows}

One may recognize that there cannot be experimentally such a thing as a truly impulsive start, although sufficiently rapid starts may be considered as producing a quasi-instantaneous motion of the body.

In theory an impulsive start may be formulated using the potential flow field as the initial condition. At time $t=0^{+}$a potential flow exists and a slip velocity (vortex sheet of zero thickness) is observed on the surface of the body.

Numerical schemes encounter difficulties in resolving the initially developed thin boundary layers associated with impulsive starts and in computing accurately quantities such as the drag coefficient that exhibits a $1 / t^{1 / 2}$ singularity. In the present formulation the impulsive start does not pose any difficulties as at time $t=0^{+}$a vortex sheet with strength $\gamma(\theta)=-2 U_{\infty} \sin (\theta)$ appears on the surface of the body. This vortex sheet is then translated into a vorticity flux as it was described in the previous section. This flux modifies the vorticity of the computational elements (of initially zero strength) residing in the neighbourhood of the boundary.

\section{Simulations of unsteady flows past a circular cylinder}

The flow behind a circular cylinder started impulsively in rotation and/or translation is investigated as a prototype of unsteady separated flows. Computations are presented for $R e=40-9500$ in order to validate our numerical method and gain some insight into the physical mechanisms present in such flows.

\subsection{Diagnostics}

The Reynolds number of the flow is defined based on the diameter of the cylinder $(D)$ (figure 1), as

$$
R e=U D / \nu,
$$

and the time $(t)$ is non-dimensionalized based on the radius $\left(R=\frac{1}{2} D\right)$ of the cylinder as

$$
T=U t / R .
$$

The principal variable of our scheme is the vorticity field, which is described as the linear superposition of the vorticity field of the individual particles. One may compute 


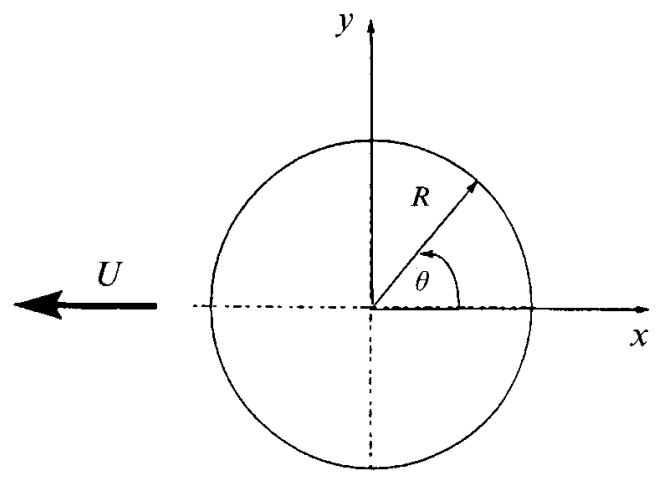

FIGURE 1. Definition sketch of the flow past an impulsively started cylinder.

diagnostics such as the streamlines, body forces and velocity field using the strength and location of those vortices.

\subsubsection{Streamlines}

The streamlines of the flow may be computed as a linear superposition of the streamlines induced by the individual particles:

$$
\Psi(x)=-\frac{1}{2 \pi} \sum_{i=1}^{N} \Gamma_{i} \log \left|x-x_{i}\right| .
$$

Note that the point-vortex streamfunction is used. This approach is dictated by the presence of the body. The use of smooth vortices near its surface would imply a nonconstant streamfunction inside the body thus violating the impermeability condition. In order to compute the streamfunction on a grid a fast algorithm is implemented. At each grid point the influence of the nearby particles is computed using (16) and multipole expansions are used to compute the influence of particles sufficiently removed from that point. This helps to reduce the cost from $O(M N)$ to $O(N+M \log N)$, where $M$ is the number of grid points on which the streamfunction is computed.

\subsubsection{Body vorticity}

The vorticity on the body is given by

$$
\omega_{\text {body }}=-\nabla^{2} \Psi
$$

As the streamfunction may be computed on a body-fitted regular grid a finite difference operator may then be applied to the Laplacian so that the vorticity is computed as

$$
\omega_{\text {body }}=-\left(7 \Psi_{0}-8 \Psi_{1}+\Psi_{2}\right) / 2 h^{2}
$$

where, if $y=0$ describes locally the body surface, then $\Psi_{0}$ is the value of the streamfunction on the body and $\Psi_{1}, \Psi_{2}$ the values at grid locations $y=h$ and $y=2 h$ respectively. Note that the high-frequency oscillations that appear on the plots of surface vorticity may be attributed to the irregular positions of the vortex particles in the vicinity of the boundary.

\subsubsection{Body Forces}

We define two independent computations for the body forces: Methods 1 and 2 that are described below. 


\section{Method 1}

The net force exerted on the body from the fluid (of unit density) may be expressed as

$$
\boldsymbol{F}_{b}=-\frac{\mathrm{d}}{\mathrm{d} t} \int_{\text {fluid }} \boldsymbol{u} \mathrm{d} \boldsymbol{x} .
$$

In order to carry out the integration over the whole domain, covering both the body and the fluid, we add and subtract the term $\mathrm{d} / \mathrm{d} t \int_{\text {body }} \boldsymbol{u} \mathrm{d} \boldsymbol{x}$ so that

$$
\boldsymbol{F}_{b}=-\frac{\mathrm{d}}{\mathrm{d} t} \int_{\text {fluid }+b o d y} \boldsymbol{u} \mathrm{d} \boldsymbol{x}+\frac{\mathrm{d}}{\mathrm{d} t} \int_{b o d y} \boldsymbol{u} \mathrm{d} \boldsymbol{x} .
$$

The velocity field inside the non-rotating body is defined by $\left.\boldsymbol{u}\right|_{b o d y}=\boldsymbol{U}_{b}$. By extending the definition of vorticity field inside the body (setting $\boldsymbol{\omega}_{b}=0$ ), we may write for the force on the body:

$$
F_{b}=-\frac{\mathrm{d}}{\mathrm{d} t} \int_{f l u i d}(\boldsymbol{\omega} \times \boldsymbol{x}) \mathrm{d} \boldsymbol{x}+A_{B} \frac{\mathrm{d} U_{b}}{\mathrm{~d} t},
$$

where $A_{B}$ denotes the area of the body. The vorticity in the wake is described in our method by the superposition of the vorticity field of the individual particles so the integral over the domain occupied by the fluid may be computed in our method by

$$
\frac{\mathrm{d}}{\mathrm{d} t} \int_{\text {fluid }} \boldsymbol{\omega} \times \boldsymbol{x} \mathrm{d} \boldsymbol{x}=\frac{\mathrm{d}}{\mathrm{d} t} \sum_{i=1}^{N} \Gamma_{i} x_{i} \times \hat{\boldsymbol{e}}_{z},
$$

where $\boldsymbol{x}_{i}=\left(x_{i}, y_{i}\right)$ is the location and $\Gamma_{i}$ the strength of the vortices in the wake. The derivative of the linear impulse in (22) is calculated numerically using a second-order central differencing scheme. The small high-frequency oscillations that are observed in some of the drag curves may be attributed to this differentiation and the fact that the remeshing procedure conserves the linear impulse of the flow but not its time derivative. Unless noted otherwise the drag coefficients presented in this paper have been computed using this method.

The expressions presented above help us also to rationalize the body forces based on the dynamics of the wake. As the wake may be described in practice by a few vortical structures, the variation with time of the location and the strength of these vortices can be used along with (22) to make inferences about the forces experienced by the body. For example, a negative (clockwise) vortex structure moving in the positive $y$-direction adds to the drag coefficient, while an increase in the strength of a positive vortex structure in the upper half-plane would reduce it.

\section{Method 2}

The drag force may also be computed as the sum of the form or pressure drag $F_{p}$ and the friction drag $F_{f}$.

The pressure drag can be determined from the flux of vorticity on the surface of the cylinder as

$$
\boldsymbol{F}_{p}=-R \int_{0}^{2 \pi} \nu \frac{\partial \omega}{\partial r} \hat{\boldsymbol{e}}_{\theta} \mathrm{d} \theta
$$

while the friction drag may be computed from the vorticity on the surface of the body as

$$
F_{f}=R \int_{0}^{2 \pi} \nu \omega \hat{e}_{\theta} \mathrm{d} \theta
$$



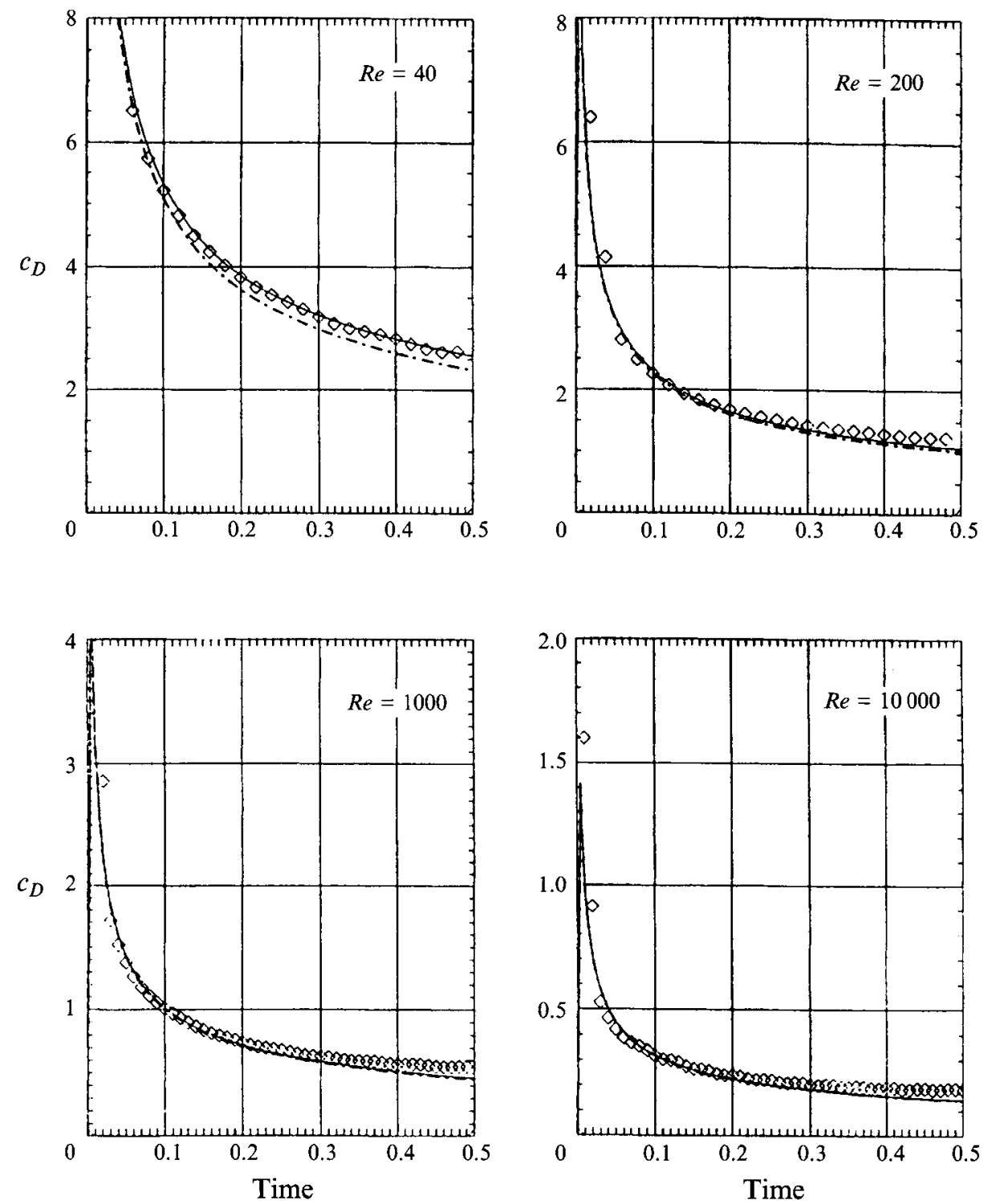

FIGURE 2. Linear plot of the early time history of the drag coefficient for an impulsively started circular cylinder. Solid line, BY; dashed line, CD; symbols, present computations.

Note that the vorticity flux is readily available in our scheme from the enforcement of the no-slip boundary condition. The vorticity on the body is computed by the numerical evaluation of the Laplacian of the streamfunction as discussed above. Hence the total drag force on the body is computed as

$$
F_{b}=F_{p}+F_{f}
$$

The drag and lift coefficients ( $c_{D}$ and $c_{L}$ respectively) of the body are given then by

$$
c_{D}=\frac{2 F_{b} \cdot \hat{e}_{x}}{U_{\infty}^{2} D}, \quad c_{L}=\frac{2 F_{b} \cdot \hat{e}_{y}}{U_{\infty}^{2} D},
$$

where $D$ is the characteristic length of the body. 


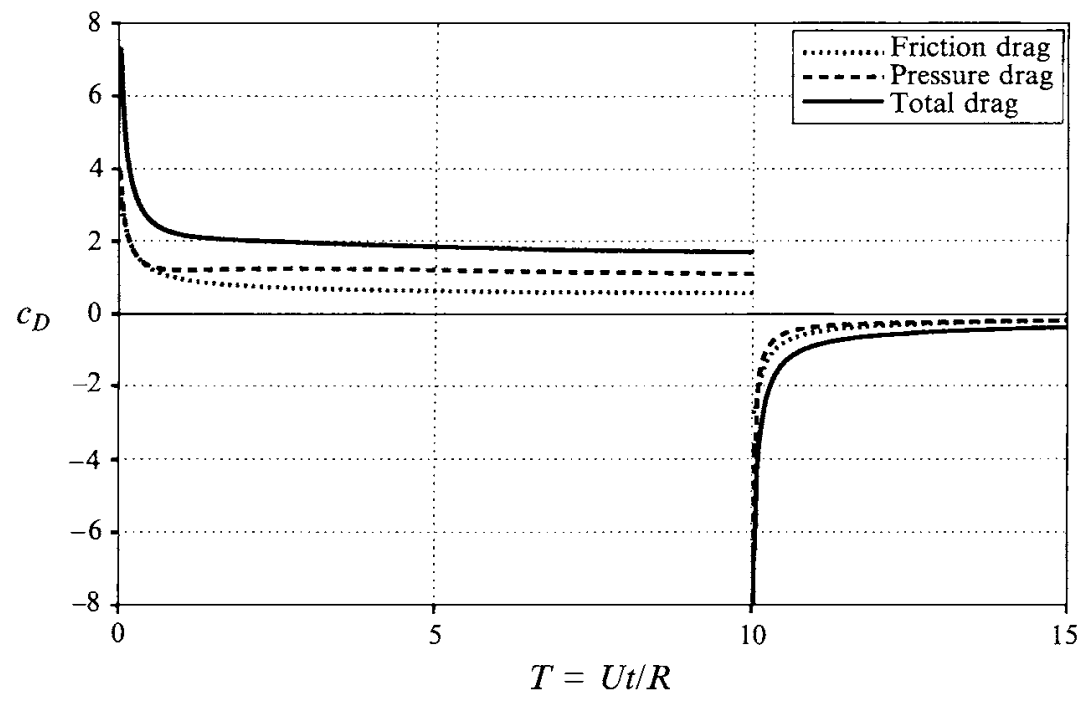

FiguRE 3. Drag coefficient for an impulsively started and impulsively stopped (at $T=10$ ) circular cylinder for $R e=40$ as computed in Method 2 .

\subsubsection{Production of circulation}

The production of circulation from the cylinder may be calculated from the known vorticity flux on its surface. For the non-rotating cylinder the total production of circulation is zero. However, we can compute the production of circulation from the upper or lower part of the cylinder in order to gain information about the circulation that is found in the vortical structures in the wake. The production of circulation is therefore computed as

$$
\frac{\mathrm{d} \Gamma}{\mathrm{d} t}=\nu \int_{0}^{\pi} R \frac{\partial \omega}{\partial r} \mathrm{~d} \theta
$$

The values of the production of circulation presented herein may be used by experimentalists to compare with the respective circulation found in the wake as well as by researchers interested in devising simplified models (such as Bryson's 1959) for the study of unsteady separated flows.

\subsection{Results}

The key features of this flow are extensively discussed in the experimental work of Bouard \& Coutanceau (1980, referred to as BC hereinafter). The flow is known to remain symmetric, for $T \leqslant 5$, for $R e$ up to 9500 . In our computation no symmetry constraint is imposed. Perturbations introduced by roundoff errors could trigger an asymmetric flow however. Our computations follow the experimental trends as the flow remains symmetric for longer times with lower Reynolds numbers.

In figure 2 the drag coefficient as computed from the present method is compared with the theoretical values of Bar-Lev \& Yang (1975, referred to as BY hereinafter) and Collins \& Dennis (1973 $a, b$, referred to as CD hereinafter). At the onset of the flow the drag exhibits a square-root singularity. The accuracy of the present vortex method is demonstrated as it is able to capture this trend for all values of $R e$. For all computations a remarkable agreement is found for times up to $T \approx 0.20$, beyond which the theoretical and computational results diverge. It is at this value of $T$ that the drag 

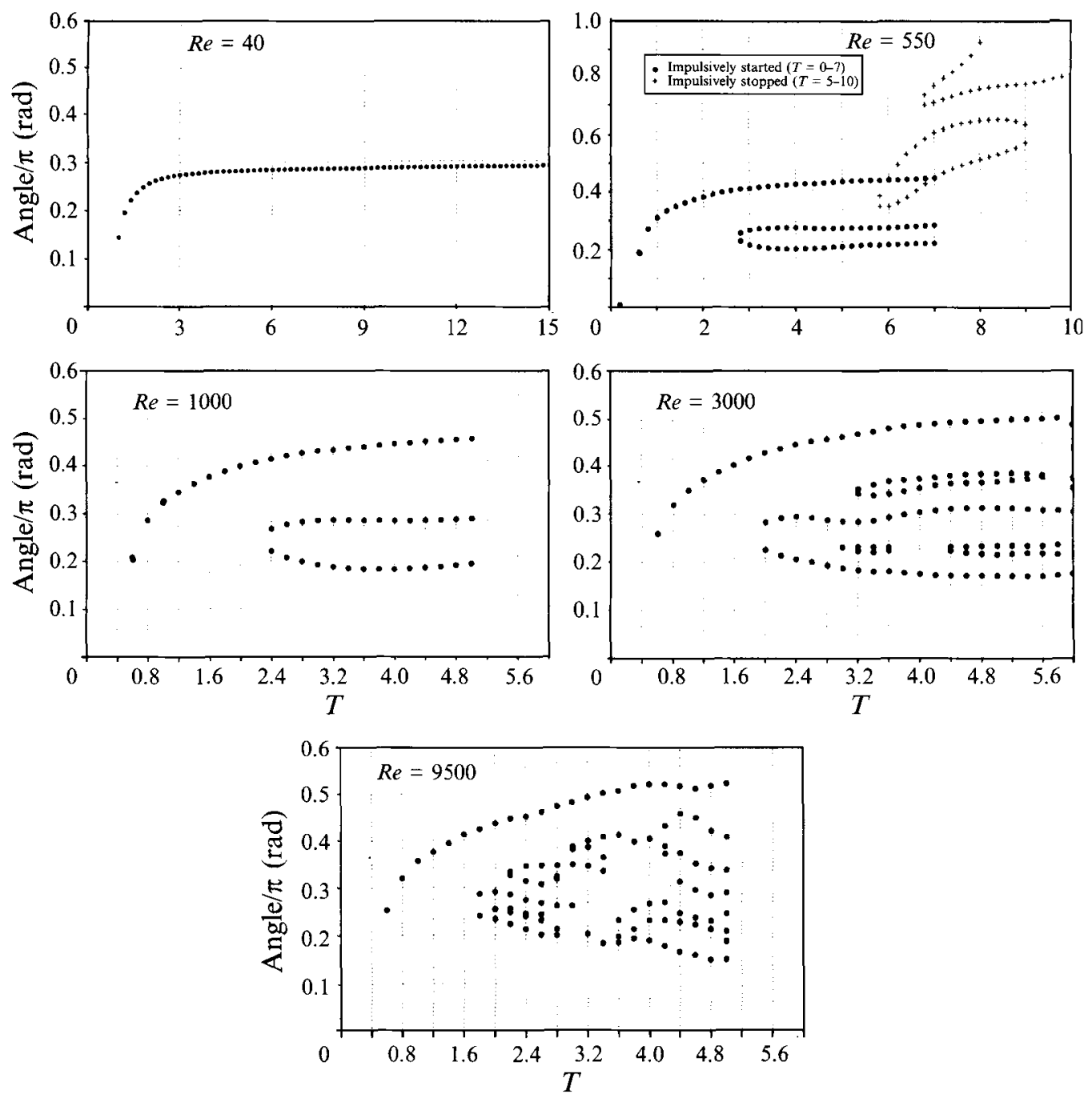

FIGURE 4. Evolution of the location of the points of zero shear stress $(\omega=0)$ with time for various $R e$.

coefficient starts to recover from the sudden drop due to the impulsive start and the effects of convection become important.

$\operatorname{Re}=40$

Vortex methods have been used in the past as a tool for the simulation of large- $R e$ flows, producing questionable results when applied to flows of large viscosity. This is mainly attributed to the problem vortex methods had in dealing with diffusion and the no-slip boundary condition. This difficulty is alleviated with the present approach and highly viscous simulations can be indeed handled.

At $R e=40$ the flow is known to reach a steady state. In the present scheme the wake is still continuing to grow at the end of the simulation. Our goal was to terminate the computation when the drag coefficient has closely approached the value it would assume after infinite time has elapsed. At $T=15$ the drag coefficient (figure 3 ) had 

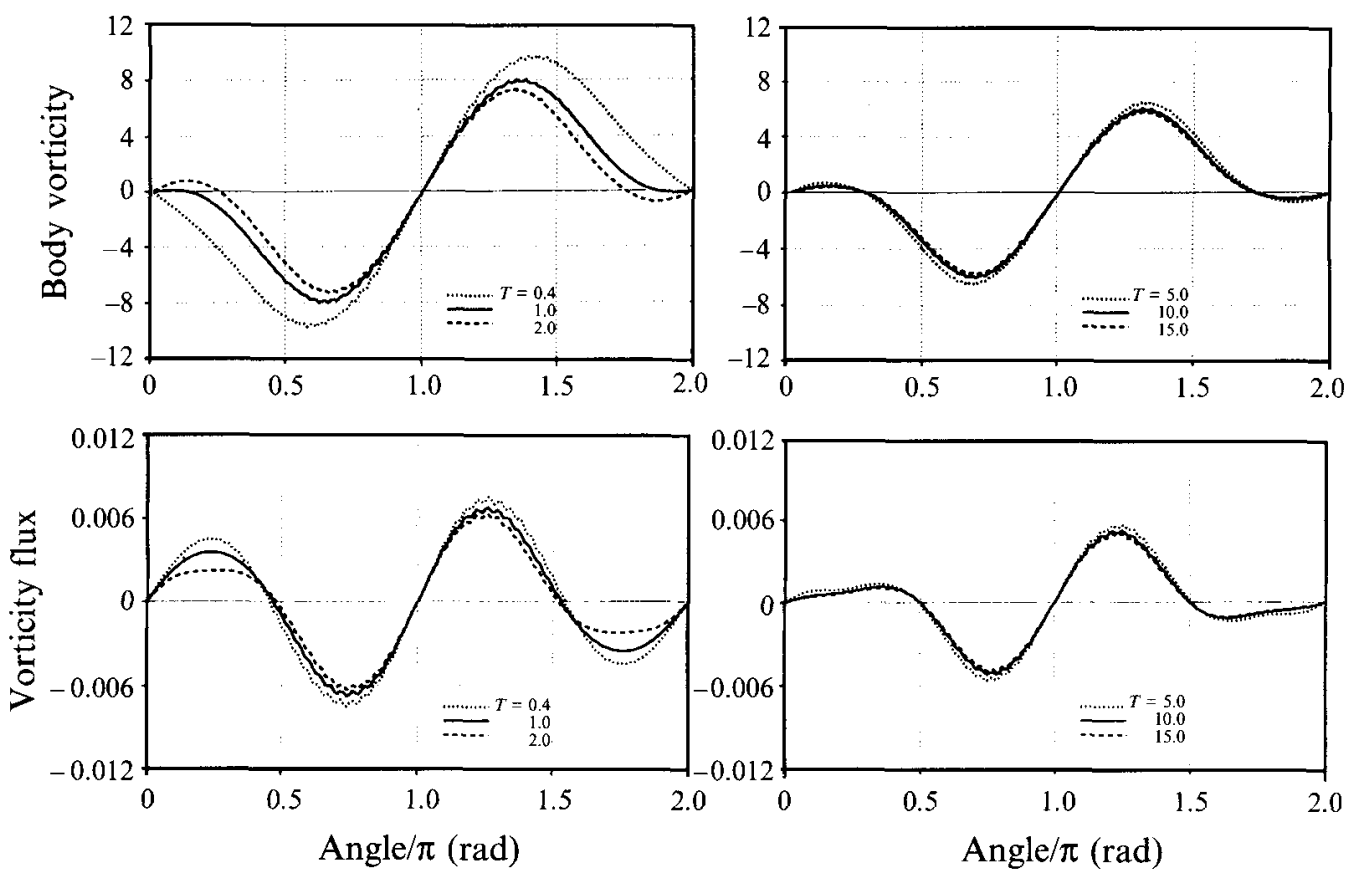

FIGURE 5. Vorticity on the surface of the body and vorticity flux $(v \partial \omega / \partial r)$ for $R e=40$.

reached a value of 1.69 , which is very close to the experimental value of 1.68 (Tritton 1959). A time step $\delta t=0.02$ was used in these simulations and at $T=15$ approximately 30000 vortices existed in the wake. An absolute non-dimensional cutoff vorticity value of $10^{-6}$ has been used. The drag (figure 3) exhibits a significant drop at the onset of the impulsive flow but does not change significantly after the appearance of secondary vorticity. Note also that initially the friction and pressure drag are equal in accordance with the predictions of BY. At later times the form drag is almost two times larger than the friction drag.

The unsteady computations exhibit the generation of the secondary vorticity at $T \approx$ 1.5. The secondary vortices initially grow and subsequently remain unchanged as they are confined by the primary vortices. Each primary vortex is passively convected by the free-stream velocity and as viscosity acts its vorticity is spread out and does not create further secondary separation effects. Although its core is diffused, no primary vortex detaches from the body as a thick shear layer continuously feeds the primary vortex uninterrupted by the secondary separation effects. As shown in figure 4, a point of zero shear (signifying separation for steady flows) appears on the surface of the cylinder, which reaches a steady state at about $52^{\circ}$ to the rear stagnation point.

Note that beyond $T=10$ the values of the vorticity and vorticity flux on the surface of the body (figure 5) remain unchanged, signifying that a steady state has been reached in our computations.

In the second stage of this simulation the cylinder is impulsively stopped. The initial condition for this flow is the vorticity field as established at $T=10$ by the previous impulsive start. As one may observe in figure 3 this impulsive stop induces a large inertial force on the body in the opposite direction to the drag. A square-root-type singularity appears in the drag because of this sudden change in the flow as well. The body force eventually decreases to zero, always remaining negative after the impulsive 

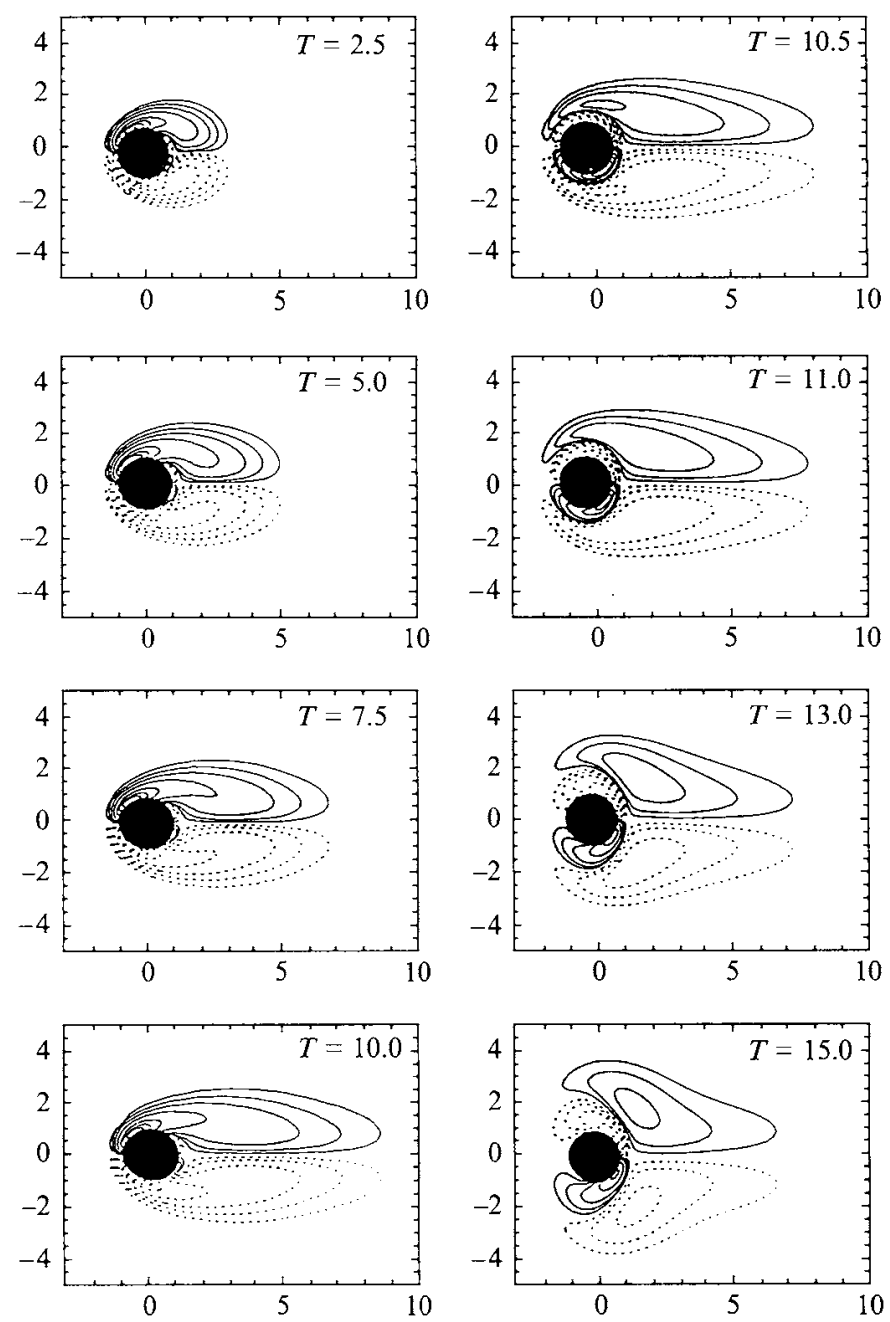

FIGURE 6. Equi-vorticity contours of an impulsively started/stopped circular cylinder for $R e=40$.

stop. In figure 6 one may observe that immediately after the impulsive stop a strong vortex layer of the same sign as the secondary vorticity develops on the surface of the body. This new layer then separates the primary vortex from the body and as it grows the magnitude of the drag is reduced. The pair of primary vortices forms a dipole that starts to move upstream. However its strength is not sufficient for it to travel far and the pair is eventually stopped as it diffuses and is stabilized by the presence of the vortex layer of opposite vorticity. At $T=15$ the vortex field has reached a configuration that essentially does not evolve further by convective effects and is subsequently eroded by diffusion.

$R e=550$

As $R e$ increases the flow structure becomes more complicated and new features of the vorticity field are exhibited. In figure 7 the streamline history of the flow field is presented. One may observe the appearance of a small secondary region as well as the development of the dividing streamline of the flow. 

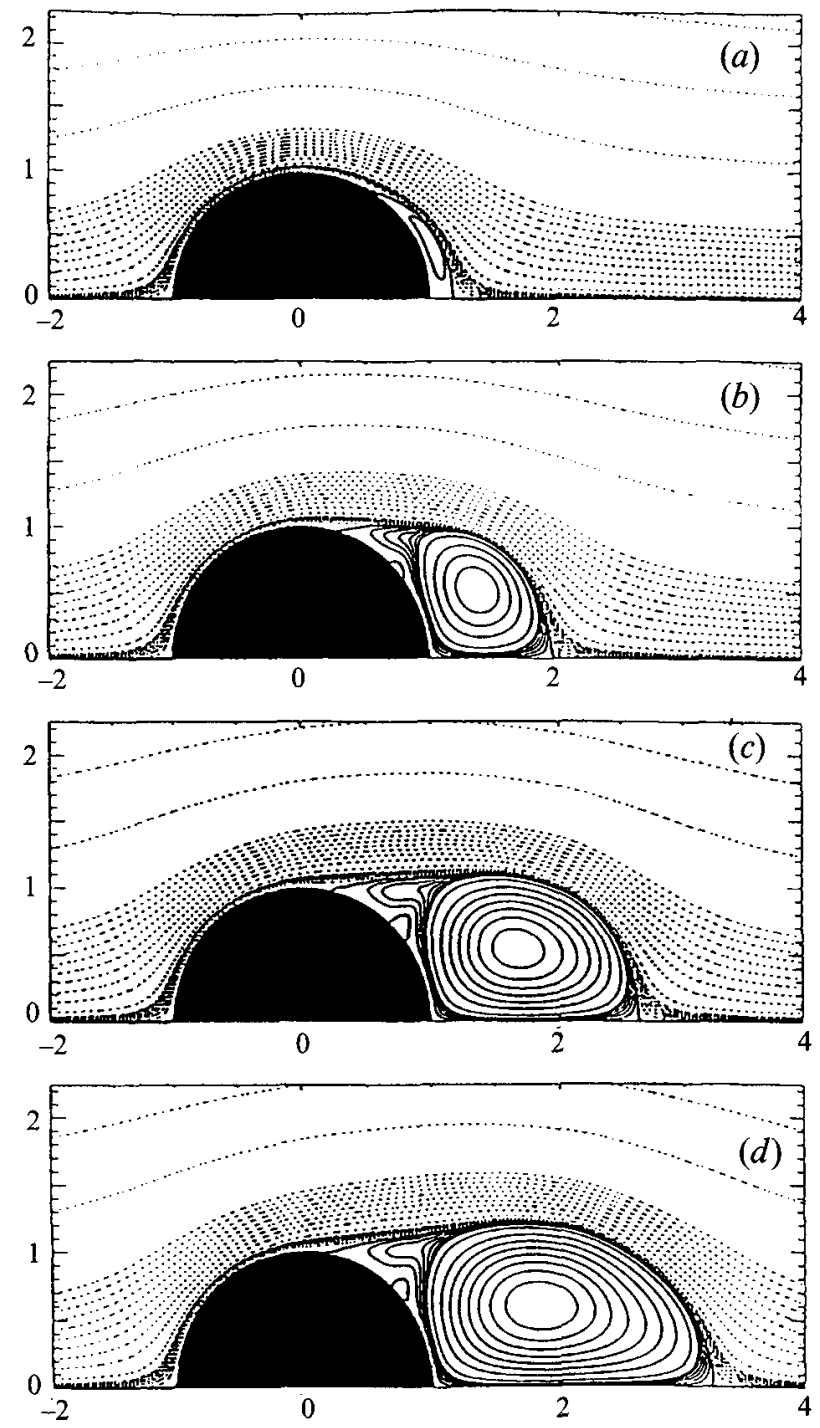

FIGURE 7. Streamline time history for an impulsively started circular cylinder at $R \boldsymbol{e}=\mathbf{5 5 0}$. (a) $T=1$, (b) $T=3$, (c) $T=5,(d) T=7$.

The development of the flow is alternatively described by the equivorticity plots of figure 8 . Although the streamline patterns show the appearance of a secondary vortex at $T \approx 3$, secondary vorticity is already visible in the vorticity plots at $T=1$. After its initial appearance the secondary vorticity remains confined and its evolution is mainly affected by the dynamics of the primary vortex. As the secondary vortex grows it penetrates the primary vortex but it is never able to reach the outer irrotational flow field. The primary vortex moves away from the body and its strength is reduced by diffusion, stopping the growth of the secondary vortex. Initially the layer that feeds the primary vortex changes angle of orientation in respect to the body but it seems that a stable configuration (the so-called $\alpha$-phenomenon in BC) is in place beyond $T=5$.

In figure 9 we present the vorticity and vorticity flux on the surface of the body. In figure 4 the evolution of points of zero shear as they appear on the surface of the 

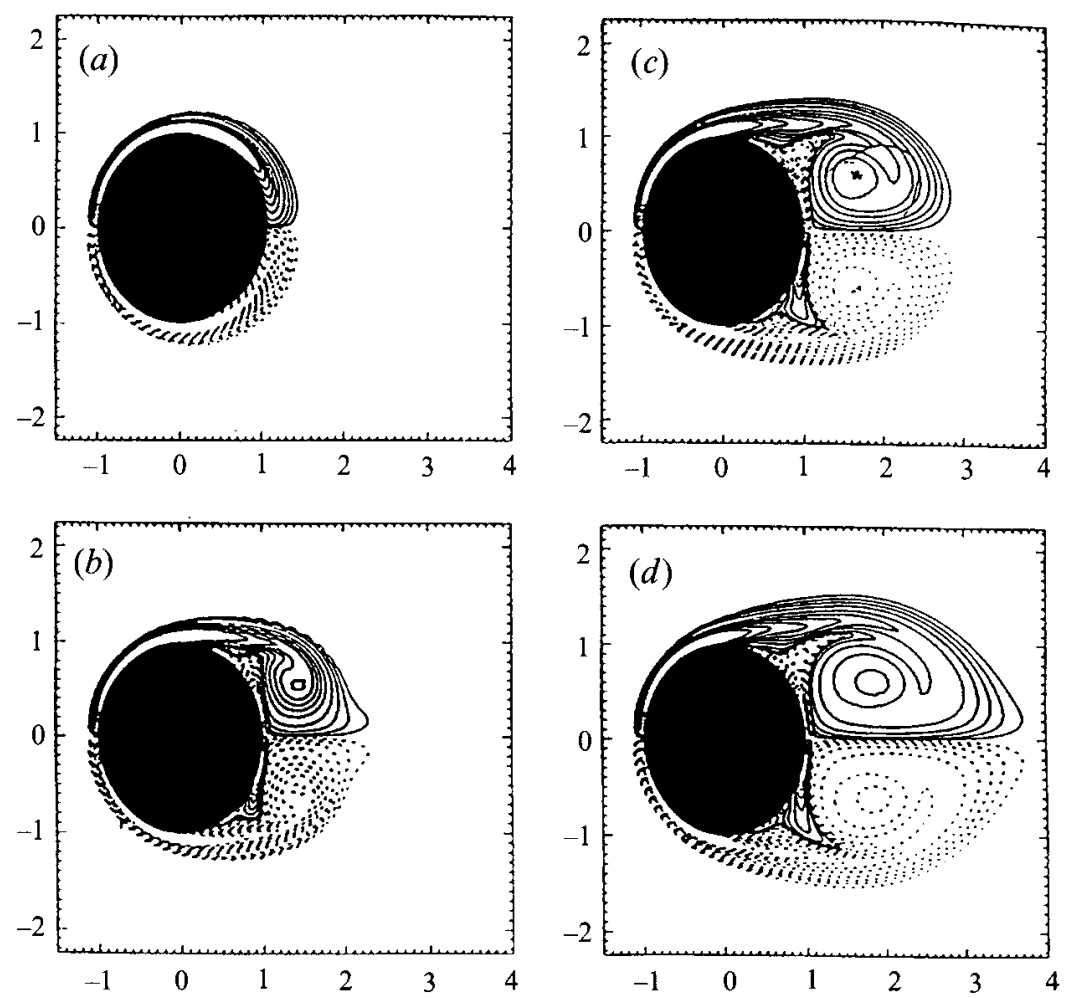

FIGURE 8. Equi-vorticity contours for an impulsively started circular cylinder at $R e=550$. (a) $T=1$, (b) $T=3$, (c) $T=5$, (d) $T=7$.
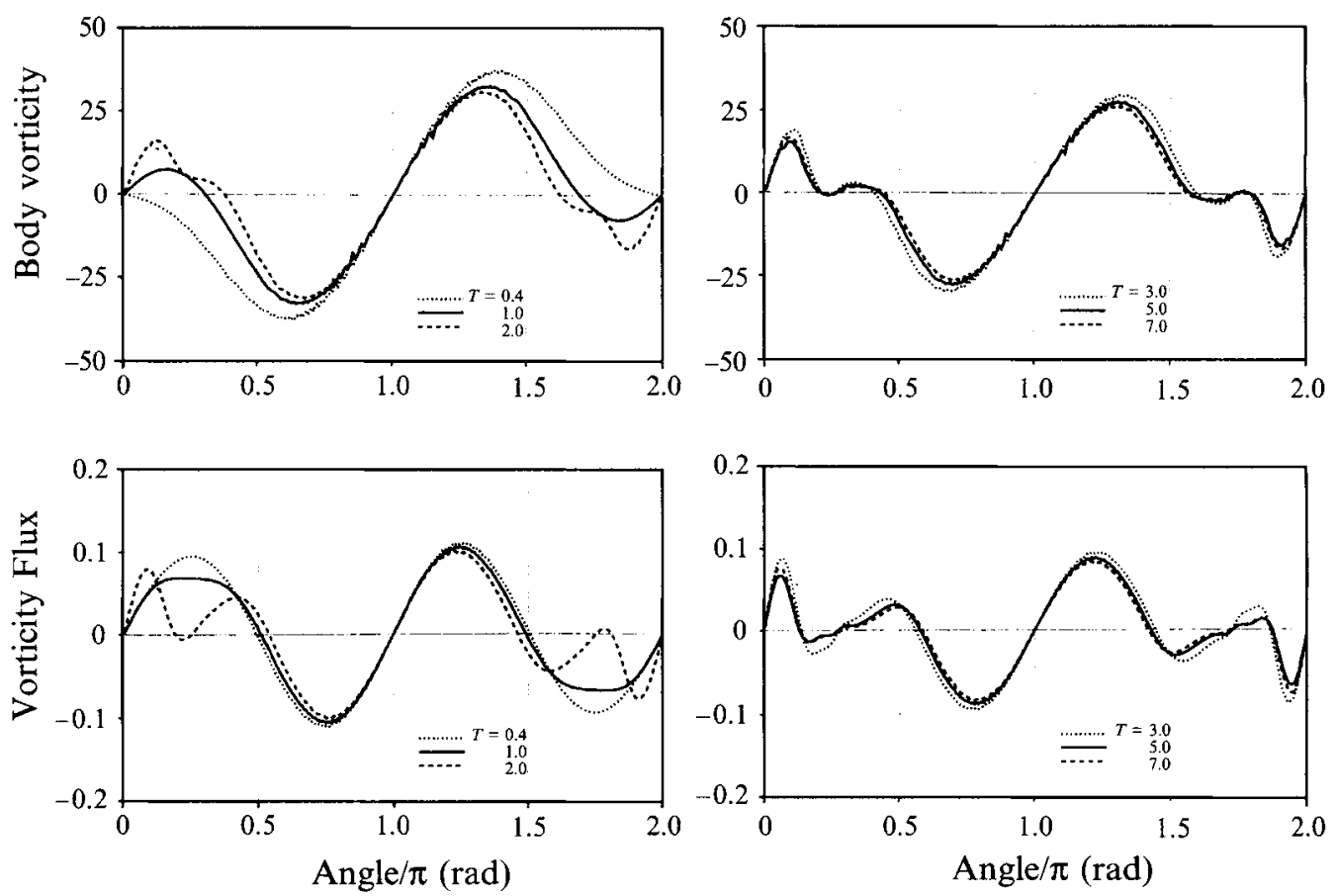

FIGURE 9. Vorticity on the surface of the body and vorticity flux $(\nu \partial \omega / \partial r)$ for $R e=550$. 

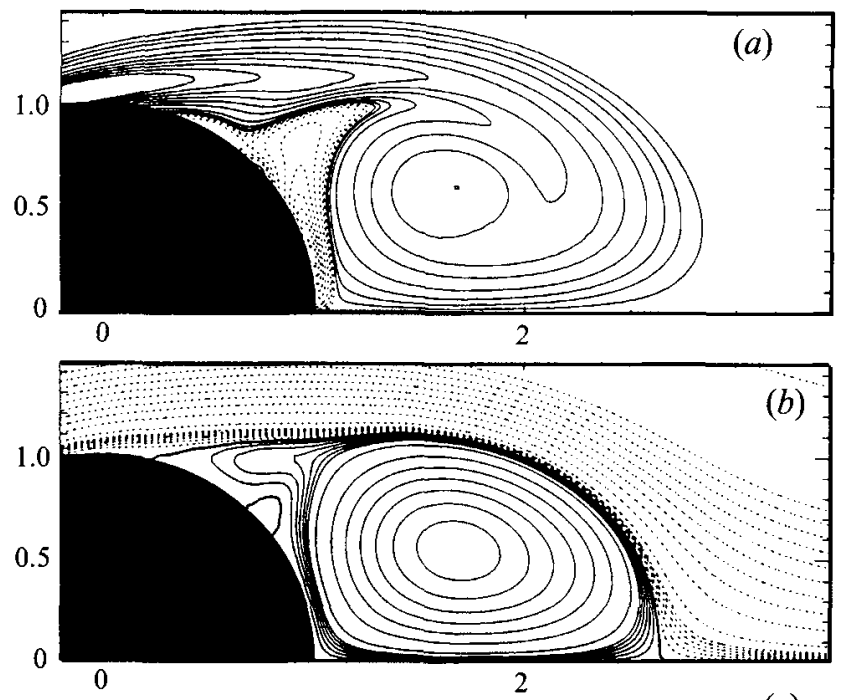

(c)

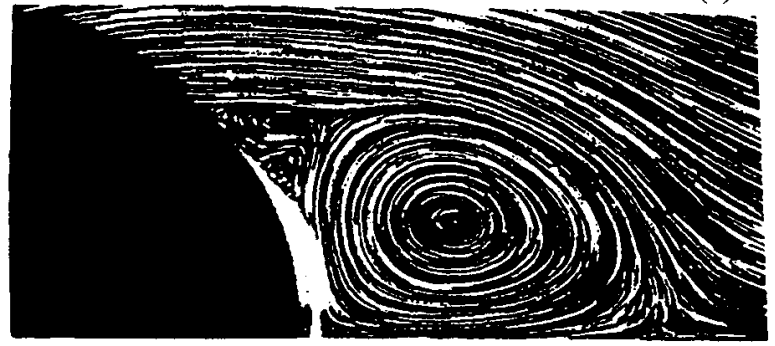

FIGURE 10. Present computational results (( $a$ ) equi-vorticity lines and $(b)$ instantaneous streamlines) and comparison with experimental results $((c)$ streaklines) of $\mathrm{BC}$ for an impulsively started cylinder for $R e=550$ at $T=5$.

cylinder is presented. From these plots one may observe the appearance and evolution of a tertiary vortical region at about $45^{\circ}$ from the rear stagnation point.

In figure 10 we compare the instantaneous streamlines obtained from these computations with the streaklines obtained by BC. The results are in good agreement. However, such good agreement in the streamline pattern has been observed in the past by other computational schemes as well. See for example figure 11, where the computational results of Chang \& Chern (1991, referred to herein as CC), Ta Phuoc Loc (1980, referred to herein as TL), and Smith \& Stansby (1988, referred to herein as SS) are presented. Note however a smaller secondary region computed by $\mathrm{CC}$ and some discrepancies with the SS computations. On the other hand, if one examines the time history of the drag coefficient obtained by different schemes the discrepancies are more pronounced. In figure 12 the drag coefficient obtained by the present numerical method is compared with the results of CC and TL as well as with the theoretical predictions of BY. The present method accurately captures the transient behaviour and is in good agreement with the results of CC. However, a large discrepancy is observed with the results of TL especially at early times. This may be attributed to the initial flow condition TL uses for the impulsively started flow. Instead of the potential flow the viscous steady-state solution at $R e=5$ has been used. Thus, initially in the computation of TL vorticity is present throughout the domain, contrary to a truly impulsive start. The moral of the story is that streamline diagnostics are very forgiving and often do 
(a)

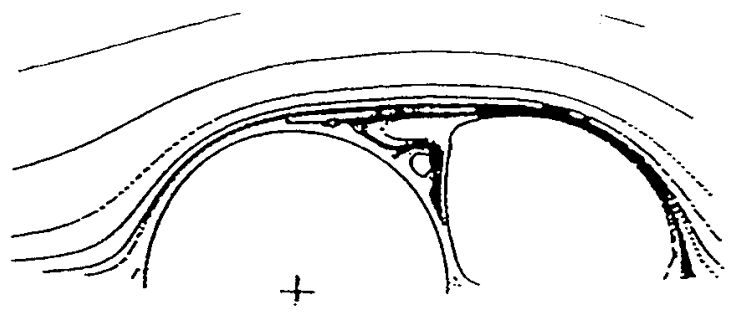

(b)

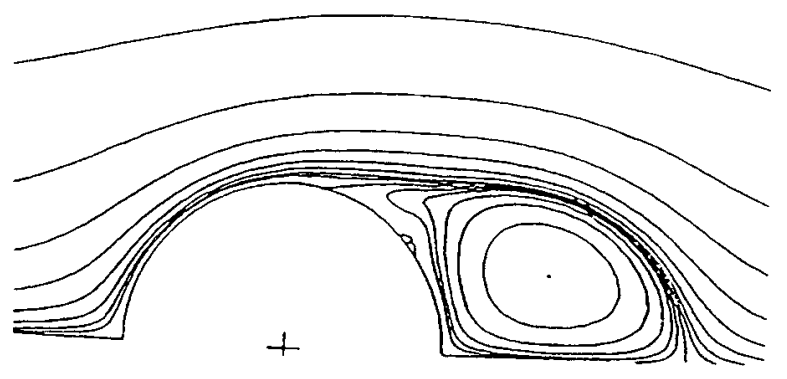

(c)

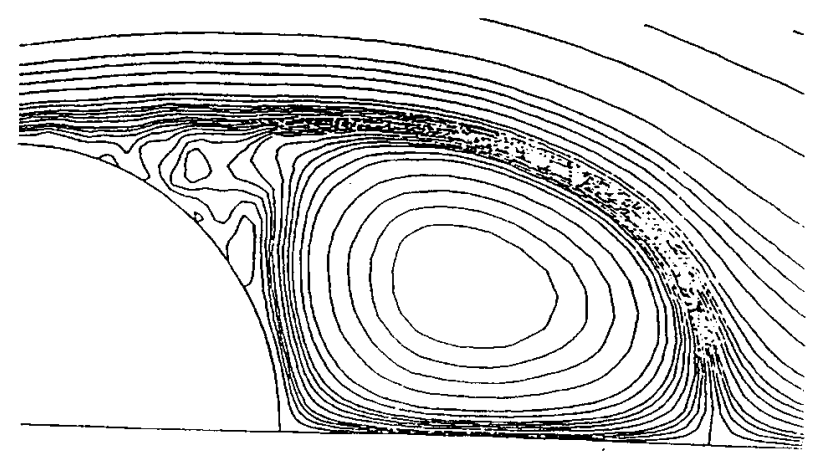

Figure 11. Computational results of $(a) \mathrm{TL},(b) \mathrm{CC}$, and (c) SS for an impulsively started circular cylinder for $R e=550$ at $T=5$.

not detect deviations from the correct solution, as they are a smooth functional of the flow, and should not be blindly trusted as indications of the validity of an approach. On the other hand vorticity fields and drag coefficients are diagnostics that are two derivatives less smooth than streamlines and their accuracy substantiates the validity of a numerical technique. Lecointe \& Piquet (1984) found that the vorticity is not accurately tracked by their high-order finite difference schemes even when a relatively consistent description of the streamlines is obtained.

The interplay of primary and secondary vorticity is manifested in the drag curve. After the initial drop in the drag the appearance and growth of the secondary vortex increases the drag coefficient as the primary vortex layer is pushed outwards. This increase reaches a maximum at $T=3$ beyond which the strength and the size of the secondary vortex are reduced whereas the primary vortex is further convected by the free stream and the drag decays to its steady state value while symmetry persists.

The drag coefficient as computed by Method 2 produced, to plotting accuracy, identical results with Method 1 . As shown in figure 13, the pressure drag accounts for about $80 \%$ of the total drag at this $R e$ after $T \approx 3$. 


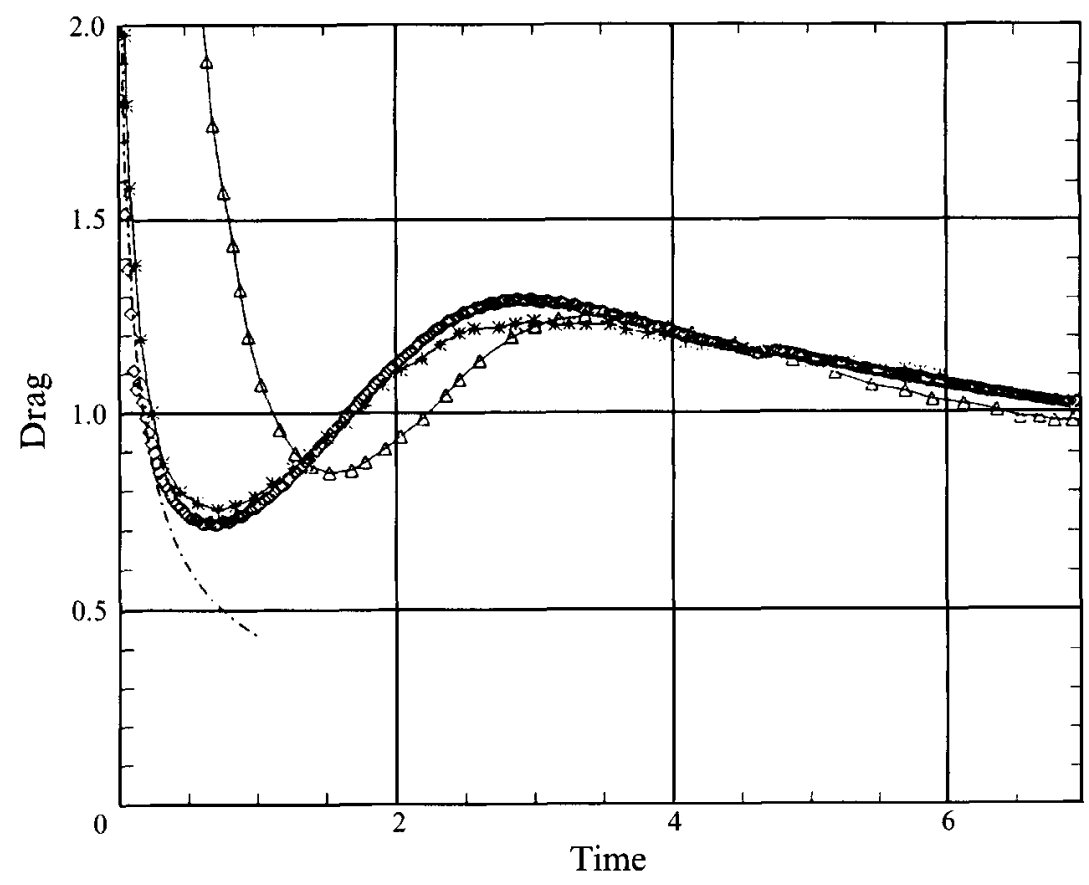

FIGURE 12. Comparison of the drag coefficient of an impulsively started circular cylinder for $R e=$ 550 as computed by several numerical schemes: $\diamond$, present; *, CC; $\triangle$, TL. Dashed line, analytical results (BY).

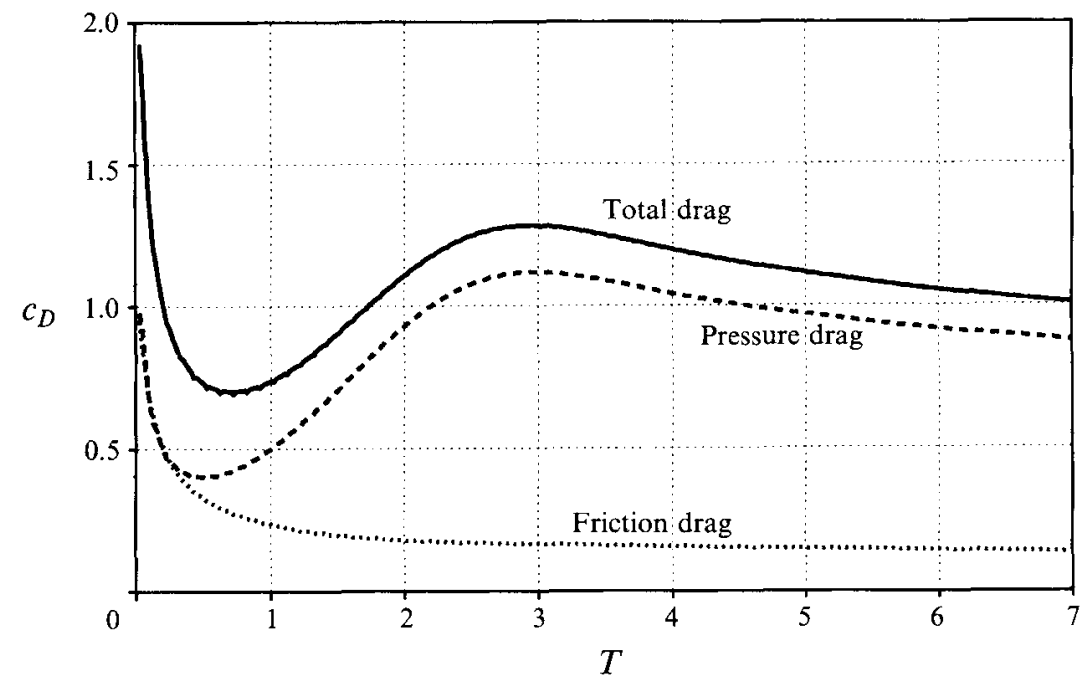

FIGURE 13. Evolution of the form and friction drag coefficient for $R e=550$.

At the second stage of this computation the flow is impulsively stopped. However, in contrast with the $R e=40$ case, the primary and secondary vortices are stronger, resulting in a more complicated flow configuration. In figure 14 the time history of the vorticity field after the impulsive stop is presented. Owing to inertial effects a strong layer of secondary vorticity is formed in the vicinity of the boundary which carries along the secondary vortex already present at the rear of the cylinder. In figure 4 we 
(a)

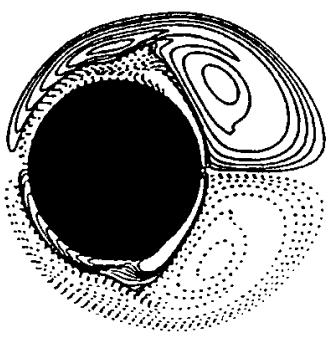

(b)

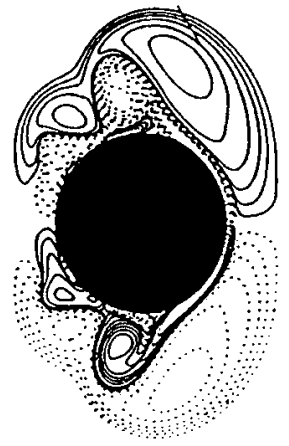

(c)

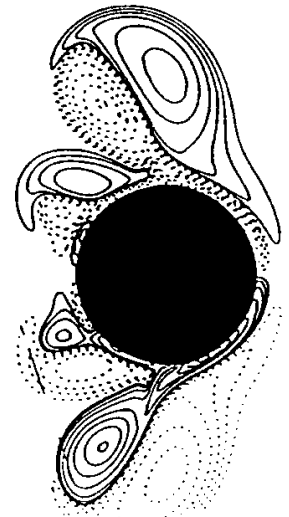

FIGURE 14. Vorticity field after the impulsive stop (at $T=5$ ) of an impulsively started circular cylinder at $R e=550$. (a) $T=6.0$, (b) $T=8.0$, (c) $T=10.0$.

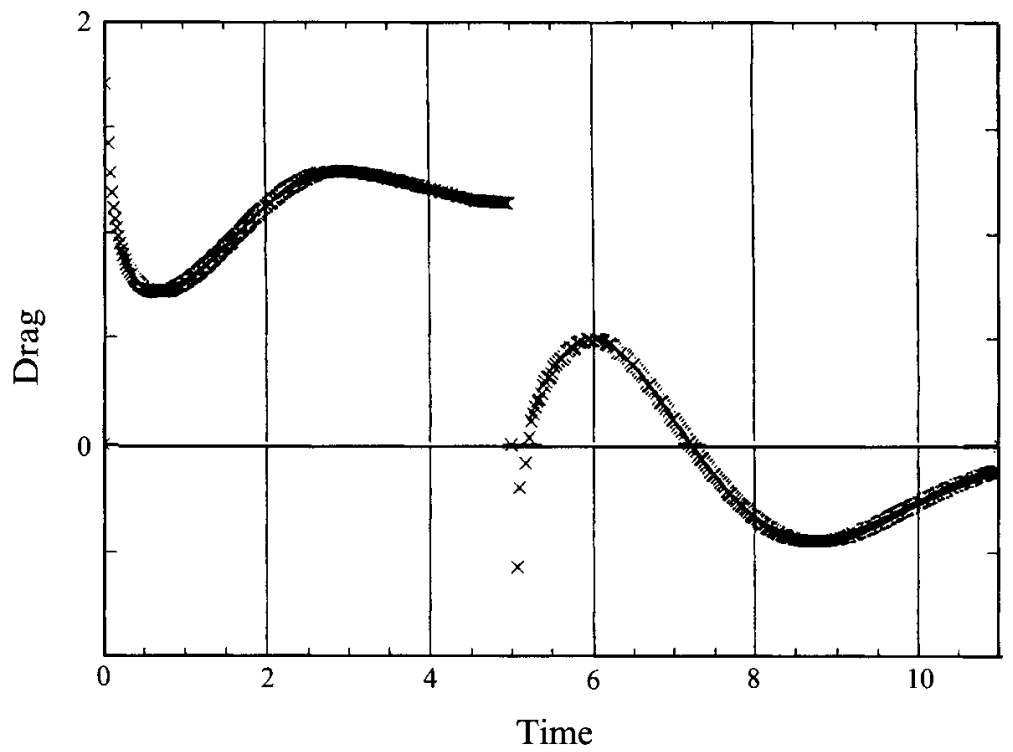

FIGURE 15. Drag coefficient for an impulsively started and impulsively stopped (at $T=5$ ) circular cylinder at $R e=550$. 
(a)

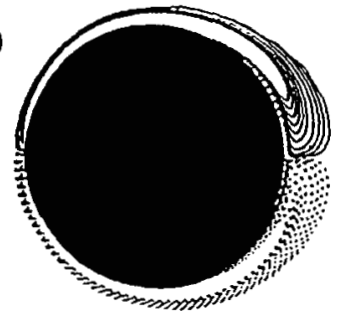

(b)

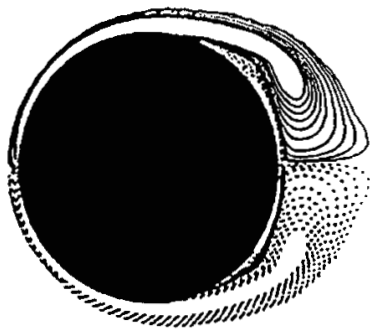

(c)

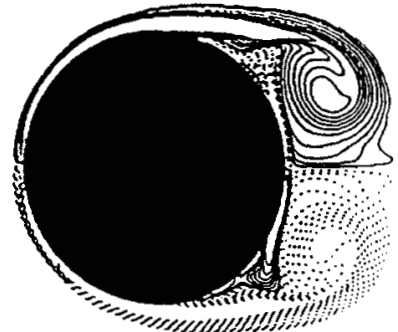

$(d)$

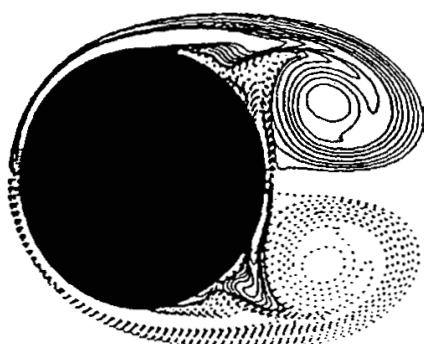

(e)

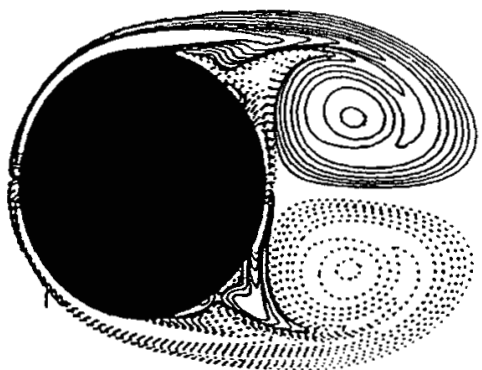

(f)

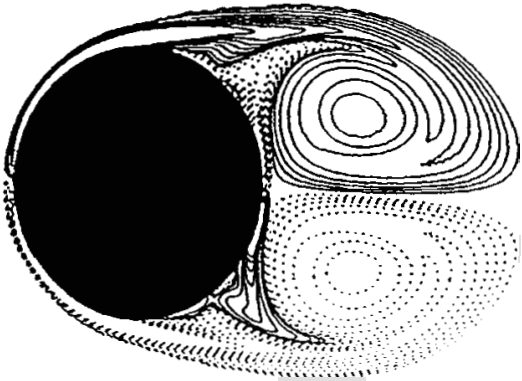

FIGURE 16. Equivorticity lines of an impulsively started circular cylinder at $R e=1000$. $(a-f)$ Times $1,2,3,4,5,6$ respectively.

do not observe any points of zero shear stress on the surface of the body between $T=5$ and 5.7 as at this stage the body is completely surrounded by this layer of secondary vorticity. Simultaneously the dipole of the primary vortices is moving upstream owing to the absence of the balancing velocity component of the free stream.

In figure 15 the drag coefficient initially becomes negative but increases rapidly enough to reach a positive maximum at $T \approx 6.0$. After this time most of the vorticity field has been carried upstream of the cylinder and tertiary vortices are formed on its surface. The secondary vortex is rearranged and eventually at $T=9$ reaches the outer flow and at the same time the drag coefficient reaches a minimum. Subsequently, new dipoles are formed around the cylinder and the vorticity field decays through diffusion, as essentially no new vorticity is generated on the surface of the cylinder, and the drag decays to zero. One may observe that although such dramatic changes occur in the vorticity field the flow remains symmetric and this symmetry is not sensitive to roundoff errors present in the computations. 

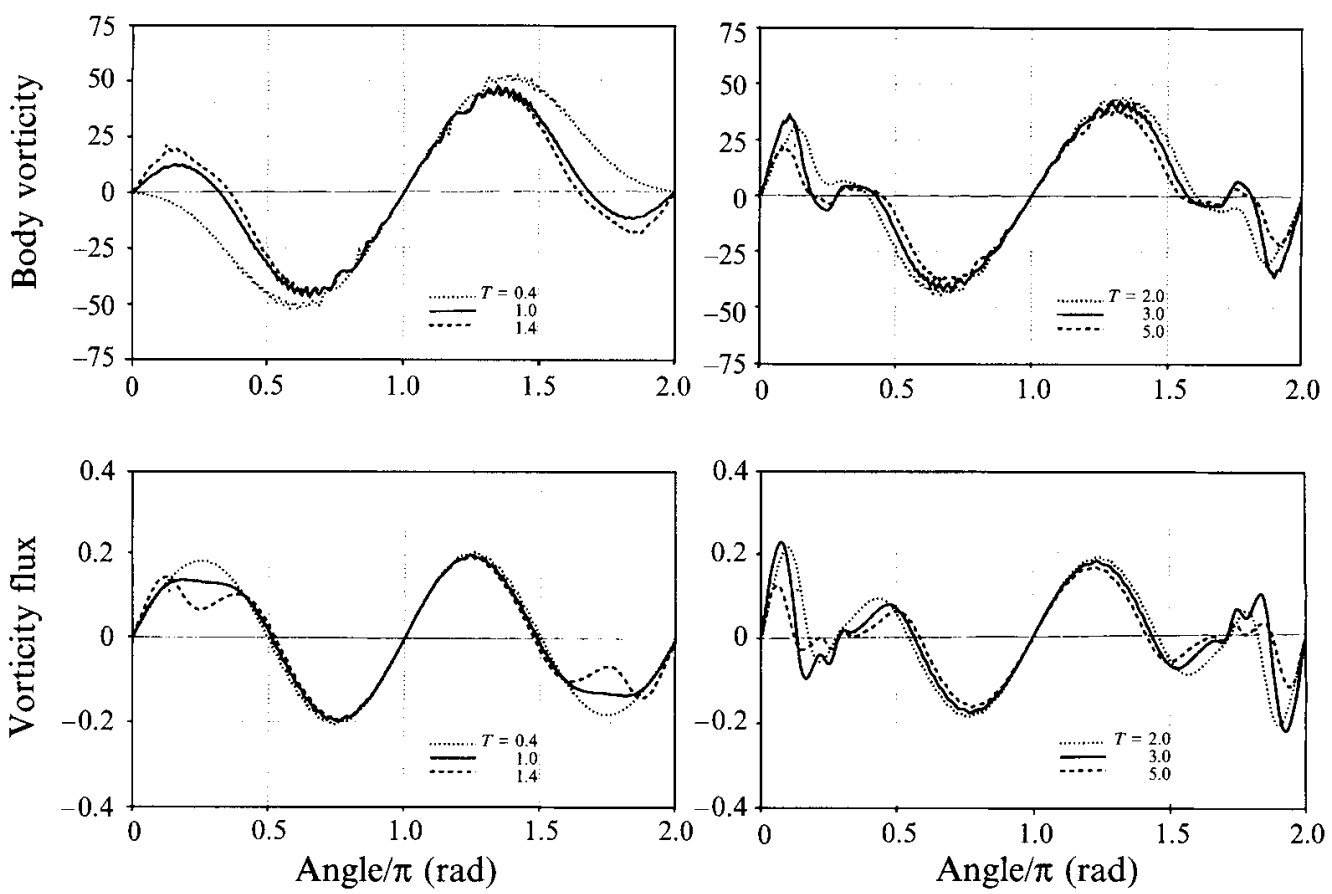

FIGURE 17. Vorticity on the surface of the body and vorticity flux $(\nu \partial \omega / \partial r)$ for $R e=1000$.

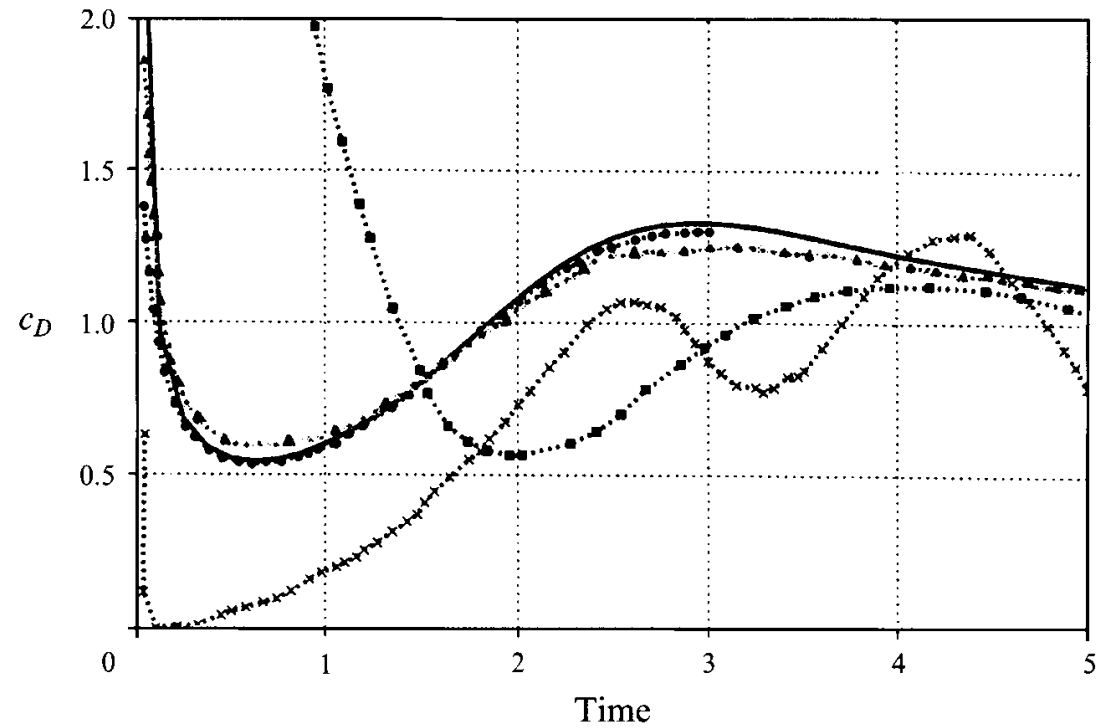

FIGURE 18. Comparison of the drag coefficient of an impulsively started at $R e=1000$ as computed

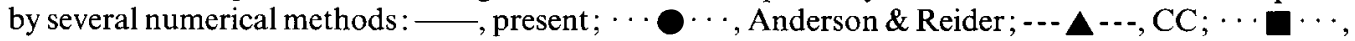
TP; $\cdots \times \cdots$, SS.

\section{$\operatorname{Re}=1000$}

The time history of the vorticity for the case $R e=1000$ is shown in figure 16 . Phenomena similar to those observed in the previous case are present here as well. The primary vortex is formed at the rear of the cylinder and the secondary vortex, although stronger in this case, remains confined by the primary vortex. The secondary vortex 


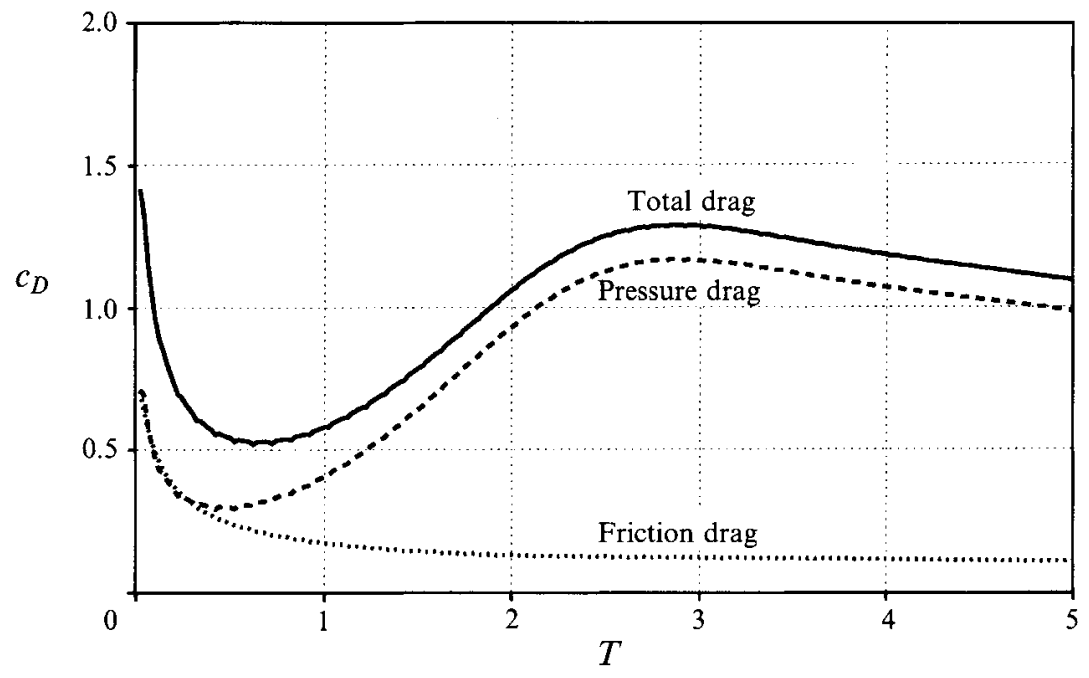

FIgURE 19. Evolution of the form and friction drag coefficient for $R e=1000$.
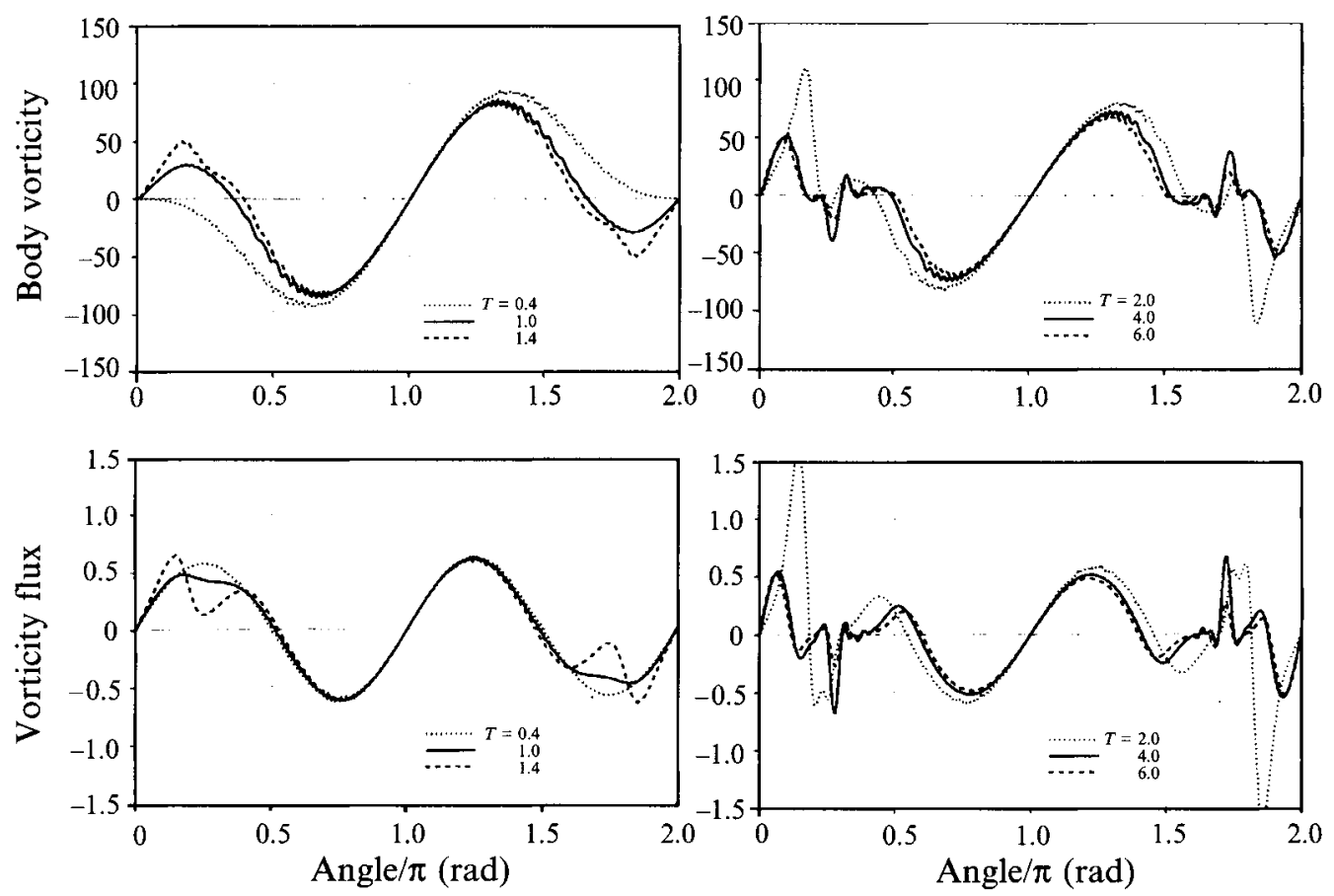

Figure 20. Vorticity on the surface of the body and vorticity flux $(\nu \partial \omega / \partial r)$ for $R e=3000$.

attempts to reach the outer flow field but is strained by the primary one before cutting the feeding link between the primary and the body. At the end of the simulation a tertiary vortex is discerned on the surface of the body. Its strength and evolution may be easily seen in figures 4 and 17 .

The parameter to observe here is the drag coefficient shown in figures 18 and 19. For this particular $R e$ several computational results are available to compare with the results of the present scheme. The present vortex method captures fairly well the initial 

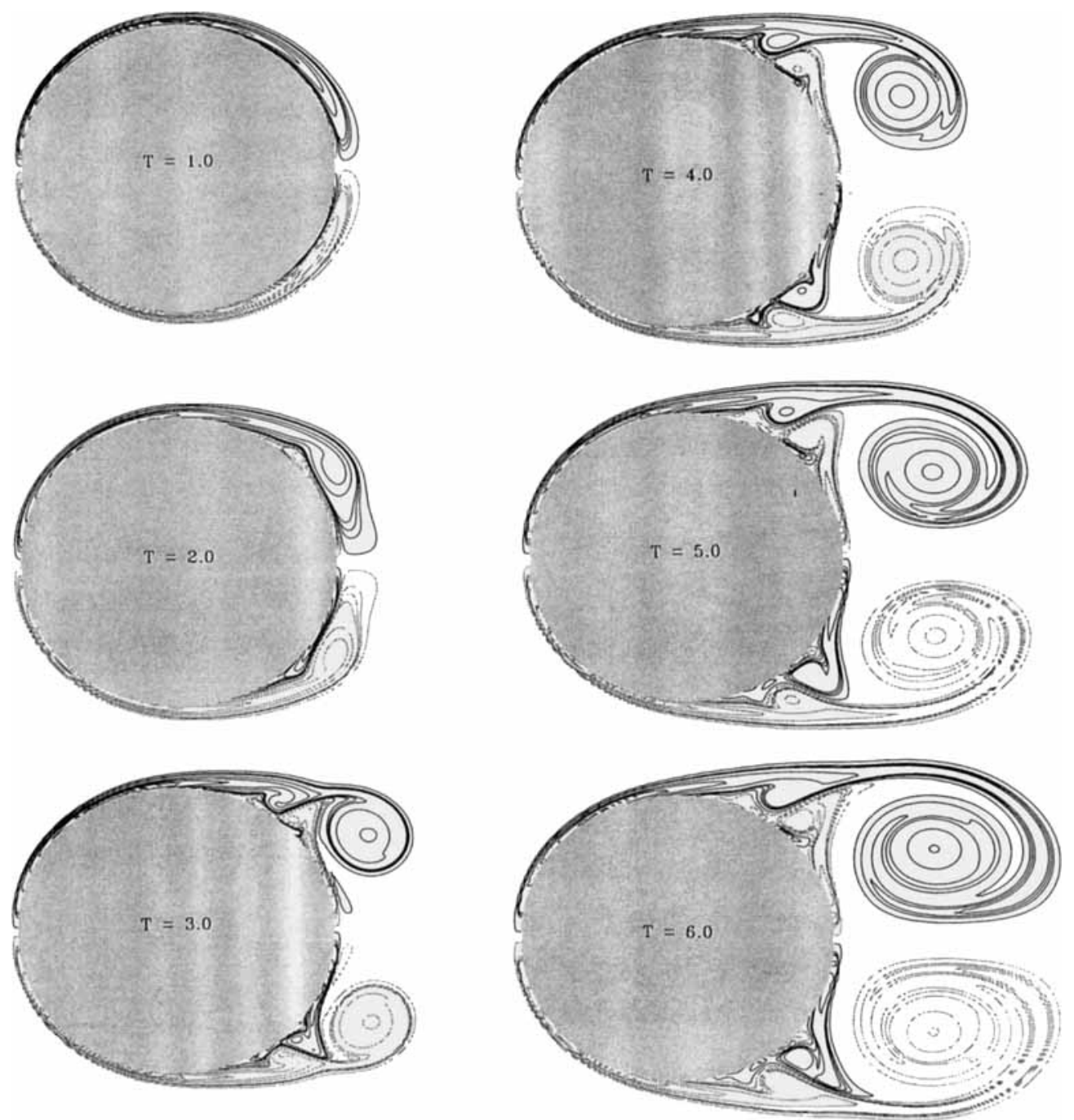

FigURE 21. Equivorticity contours of an impulsively started circular cylinder at $R e=3000$.

square-root singularity of the drag coefficient and is in agreement with the theoretical results of BY. The present results are also in very good agreement with those from the computations of Anderson \& Reider (1993). They used a fourth-order finite difference scheme and a grid of $512 \times 512$ to capture the evolution of the flow up to $T=3.0$. The agreement with the method presented in $\mathrm{CC}$ is still satisfactory although some discrepancies appear around the minimum and maximum of this curve (when vorticity is most active). These discrepancies may be attributed to the relative low resolution $(256 \times 200)$ of their simulations and the additional viscous dissipation present in their scheme that reduces the actual strength of the vortices.

Large discrepancies appear, however, between the results of the present method and those presented by TL and SS. The effects of the initial condition selected by TL become more pronounced at this higher $R e$. The flow of TL seems to never recover to that corresponding to an initially impulsively started cylinder. On the other hand the scheme used by SS also presents an erratic behaviour. The initial transient behaviour is not captured (even giving zero and negative drag), demonstrating the problems of the 


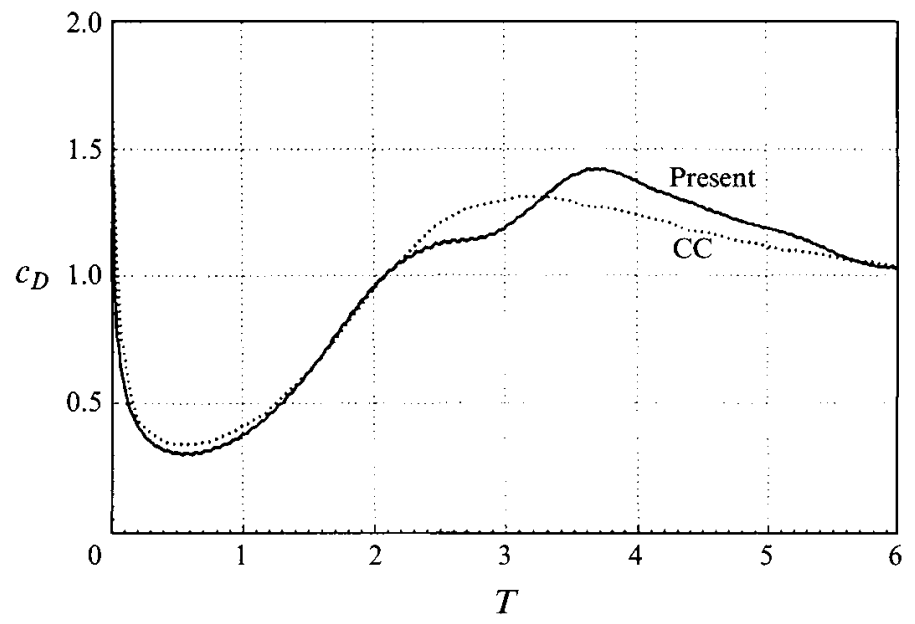

FIGURE 22. Drag coefficient of an impulsively started cylinder at $R e=3000$.

algorithm in simulating vorticity generation due to solid walls and possibly the low convergence rate of the random walk approach to model viscous effects. At later times the drag coefficient oscillates, signifying dynamics not present in any other computation.

$R e=3000$

At this Reynolds number a series of new separation phenomena appears. The primary vorticity that is formed at the rear of the cylinder is stronger than in lowerReynolds-numbers cases, thus producing a strong adverse pressure gradient at the rear of the cylinder. A region of strong secondary vorticity is thus produced at the rear of the cylinder, reaching a peak at about $30^{\circ}$ from the rear of the cylinder at $T=2$ (figure 20 ). This secondary vorticity induces in turn tertiary vortical regions of the surface of the cylinder. The evolution of this cascade of points of zero shear stress is shown in figure 4.

The secondary vorticity that is generated on the surface of the cylinder attempts to penetrate the primary vortex between $T=2.0$ and 3.0. Simultaneously the drag coefficient maintains a constant value as the feeding of the primary vortex is weakened. However, a second vortex is formed by the separating shear layer that again suppresses the secondary vorticity at $T=3.0$ (figure 21 ). The feeding of the primary vortex is reestablished and the drag coefficient increases again (figure 22). As a result of the increased strength of the primary vortex the secondary vorticity gains strength again, reducing the drag experienced by the body for $T \geqslant 3.7$. As shown in figure 23 , the friction drag (after the initial start) maintains a small constant value while the pressure drag as determined by the interplay of the vortical structures with the body accounts for about $95 \%$ of the total drag.

Note that the history of the drag coefficient as computed by the present method is not in agreement with the results of CC. However, they use the same grid of $128 \times 200$ as for their simulations at lower $R e$. In the present simulations about an order of magnitude more computational elements $(N \approx 300000)$ (figure 24$)$ are present at $T=$ 6.0 to resolve all the phenomena present in the flow. Moreover the Lagrangian character of the method enables us to concentrate our resolution where the 'action' is, thus automatically capturing phenomena such as the bulge formation of the secondary vorticity and its stripping by the primary vortices. 


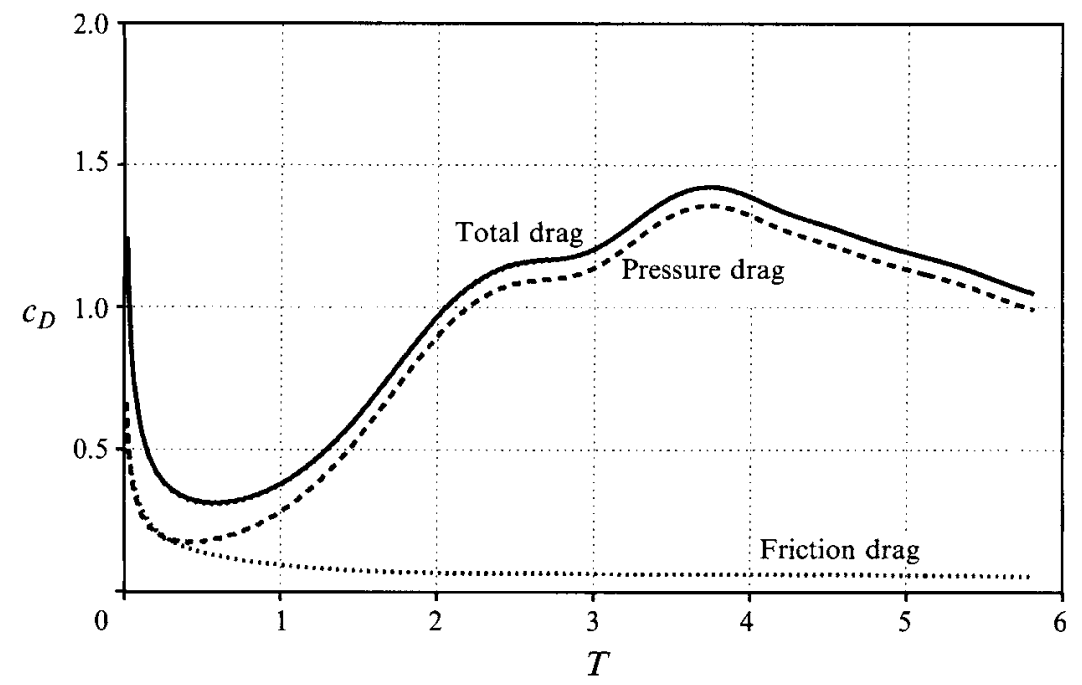

FIGURE 23. Evolution of the form and friction drag coefficient for $R e=3000$.

$R e=9500$

The case $R e=9500$ is the highest $R e$ for which simulations are presented in this work and is by far the most interesting and challenging one. The time step used is $\delta t=0.01$, the cutoff vorticity limit is kept to $10^{-5}$ and at the end of the simulation at $T=6.0$ more than a million vortices are used (figure 24) to capture all the scales present in the flow.

In figure 25 we present a comparison with the experimental results of BC. However, a more vivid description of the flow is presented in terms of the vorticity field in figure 26. Initially a very thin layer of vorticity resides around the cylinder as it is introduced by the initial potential flow. This thin layer continues to grow while maintaining a relatively invariable form. At the same time the square-root singularity is observed in the drag coefficient. However, after $T \approx 1.5$ a bulge of secondary vorticity forms at $\theta \approx 50^{\circ}$ that modifies the dynamics of the wake. As it is also stronger it rises rapidly, penetrates the feeding vortex sheet of the primary vortex at $T \approx 2.25$, and reaches the outer flow. A recent review by Doligaski, Smith \& Walker (1994) discusses thoroughly this eruptive procedure and provides a complete account of past research of the phenomenon of unsteady separation.

This eruption of the boundary layer of the flow past an impulsively started cylinder was first discussed by Van Dommelen \& Shen (1980). According to them this bulge of vorticity is the signature of unsteady separation. However, they predict that such a phenomenon would occur at an angle of about $70^{\circ}$ from the rear stagnation point for infinite $R e$. This discrepancy of about $20^{\circ}$ with present observations may be attributed to the finite (although large) $R e$ of the simulation. Indeed this secondary vortex is formed at a much larger angle from the rear stagnation point than at lower $R e$. In addition it is possible that the unsteady boundary layer theory is not applicable for such late times.

The primary vortex (V1) subsequently rolls up and eventually detaches from the body, at $T \approx 2.50$, carrying along a part of the secondary vortex. As the secondary vortex is severed its strength reduces and a new positive bulge of vorticity, created by the shear layer, comes to suppress it. The new vortex (V2) is formed at a yet higher angle, extracting some positive vorticity from the body as well so that the secondary vortices 


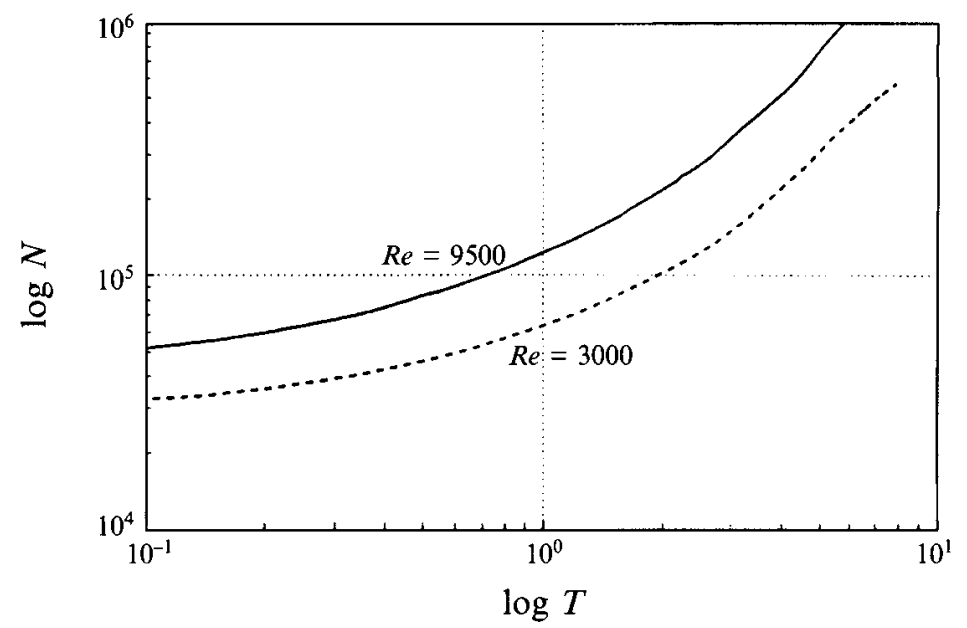

FIGURE 24. The number of computational elements versus time for an impulsively started cylinder at $R e=9500$ compared to $R e=3000$.

have a wave-like shape. As the new vortex moves downstream, it reaches the primary vortex and merges with it, re-establishing the link between the primary vortex and the body.

The interplay of the primary and secondary vortices does not remain unnoticed by the drag coefficient (figure 27). The drag, after its initial drop, increases as the primary vortex continues to be displaced by the secondary vorticity. However, at $T \approx 2.0$ when the secondary vortex reaches the outer flow and the primary vortex is detached (figure 26), the drag remains relatively constant for a short time and continues to increase when the feeding of the primary vortex is re-established. As the Re increases, the drag force is mainly attributed to the pressure drag. Also, at this $R e$ we observe for the first time a slight discrepancy between the drag coefficients computed by Methods 1 and 2 . This discrepancy may be attributed to the remeshing procedure as well as to the fact that the computation and subsequent distribution of the vorticity flux deteriorates with the increase of $R e$.

The present results are in good agrement with the high-resolution simulations of Anderson \& Reider (1994) and Wu et al. (1995) (figure 28). They use finite difference schemes with grids of $(2046 \times 256)$ and $(512 \times 300)$ respectively to conduct simulations up to $T=3.0$. Note that for the same $T$ the present scheme uses about 350000 vortices. The present results disagree with those presented by CC. More specifically the scheme of $\mathrm{CC}$ does not predict the drag plateau that appears at $T \approx 2.0$. It seems that the relatively low resolution $(256 \times 200)$ and the additional numerical diffusion present in that scheme prevent it from capturing subtle details of the flow.

At subsequent times $(T=3-6)$ the phenomena discussed above are more pronounced and enable us to validate our observations on the role of the vortical structures in the drag coefficient experienced by the body. One may observe a series of tertiary vortical phenomena appearing on the surface of the cylinder and a repetition of the unsteady separation procedure at smaller scales. This appearance and evolution of regions of tertiary vorticity on the surface of the cylinder is depicted in figures 4 and 29 , as well as in the streamline history of the flow (figure 30 ).

At $T=3.25$ we observe the merging of V1 and V2 while at the same time the link with the separating shear layer is weakened. However, the secondary vorticity is not able to penetrate into the outer flow as the primary vortex nears the body and 
(a)

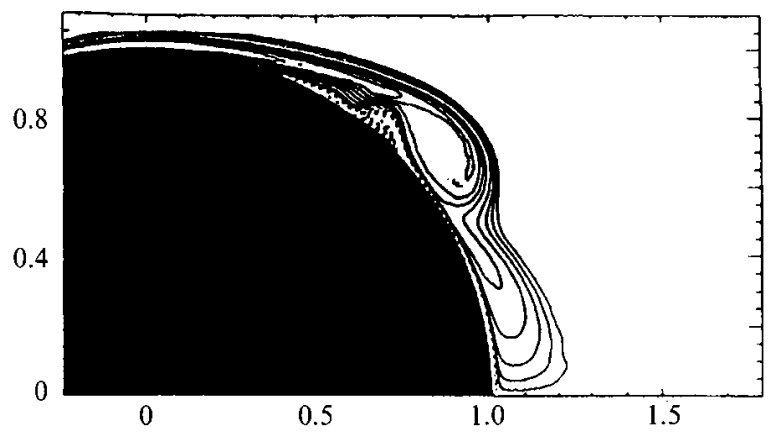

(b)

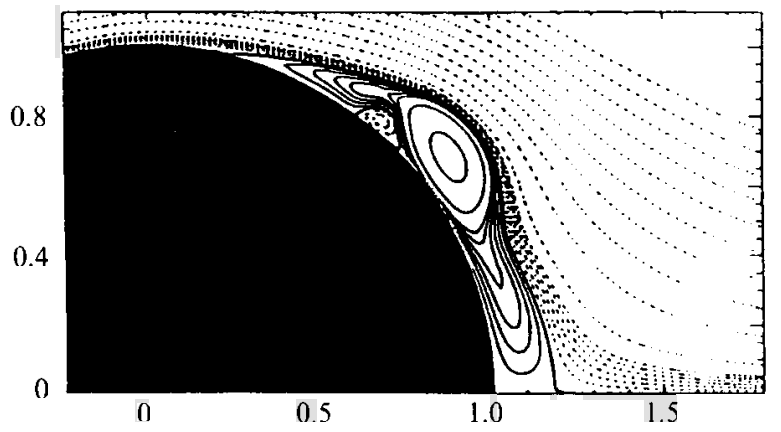

(c)

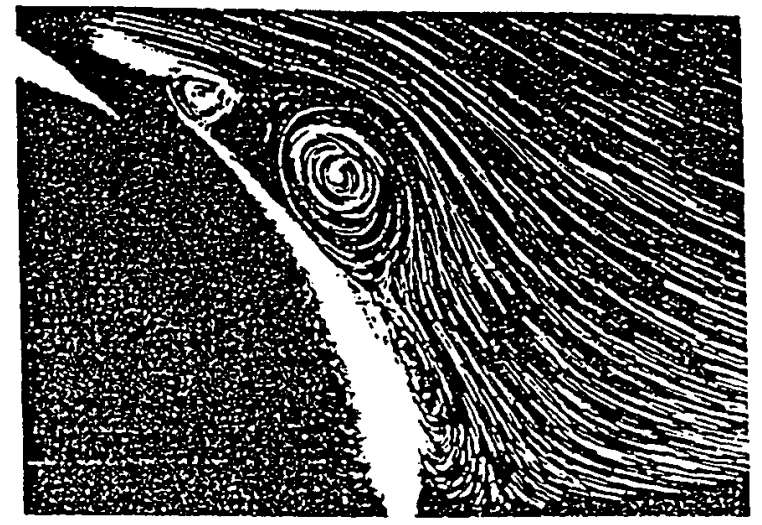

FIGURE 25. (a) Equivorticity lines and (b) instantaneous streamlines from computations and $(c)$ experiments $(\mathrm{BC})$ for $R e=9500, T=2.0$.

accumulates the rest of the same-sign vorticity over the secondary vortex. At the same time the link of the primary vortex to the shear layer is re-established and as the merged vortices are in the vicinity of the body the drag reaches its maximum value. However, the primary vortex continues to extract secondary vorticity from the surface of the body and as a third vortex (V3) is shed from the shear layer the drag coefficient starts to decrease $(T=4.00)$. This effect appears more pronounced at $T=4.25$ and $T=4.50$ when the secondary vorticity has reached the outer flow. One may observe then a series of alternate-sign vortices bursting from the surface of the body. When the secondary vorticity reaches the outer fluid it is elongated and subsequently severed by the main vortex. This enables the shear layers to roll over it again while increasing the drag coefficient. The sequences $T=5.50,5.75,6.00$ show in a milder manner the primary-secondary vorticity interplay and its effect on the drag coefficient.

Finally in figure 31 one may observe the production of circulation from the surface 

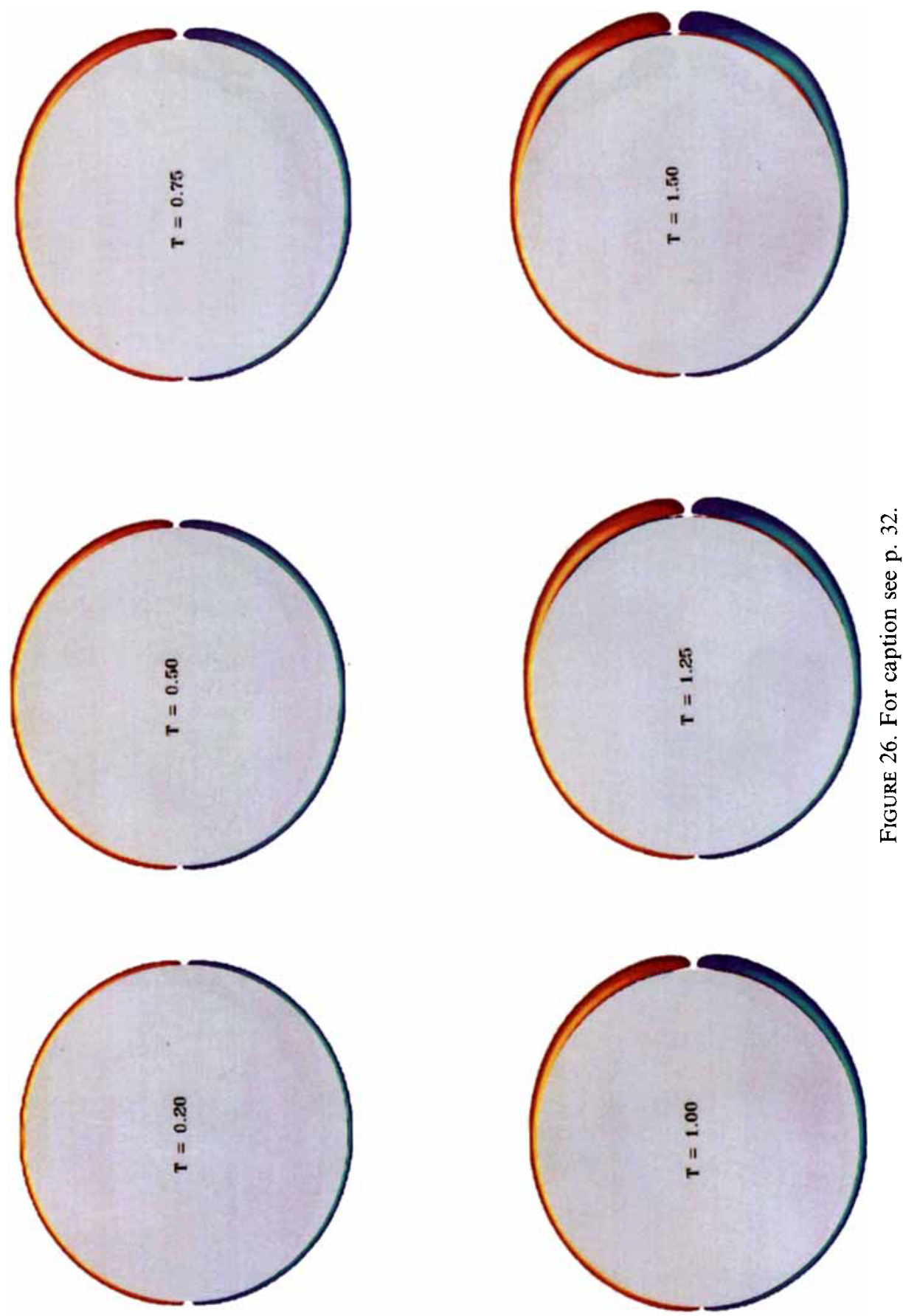

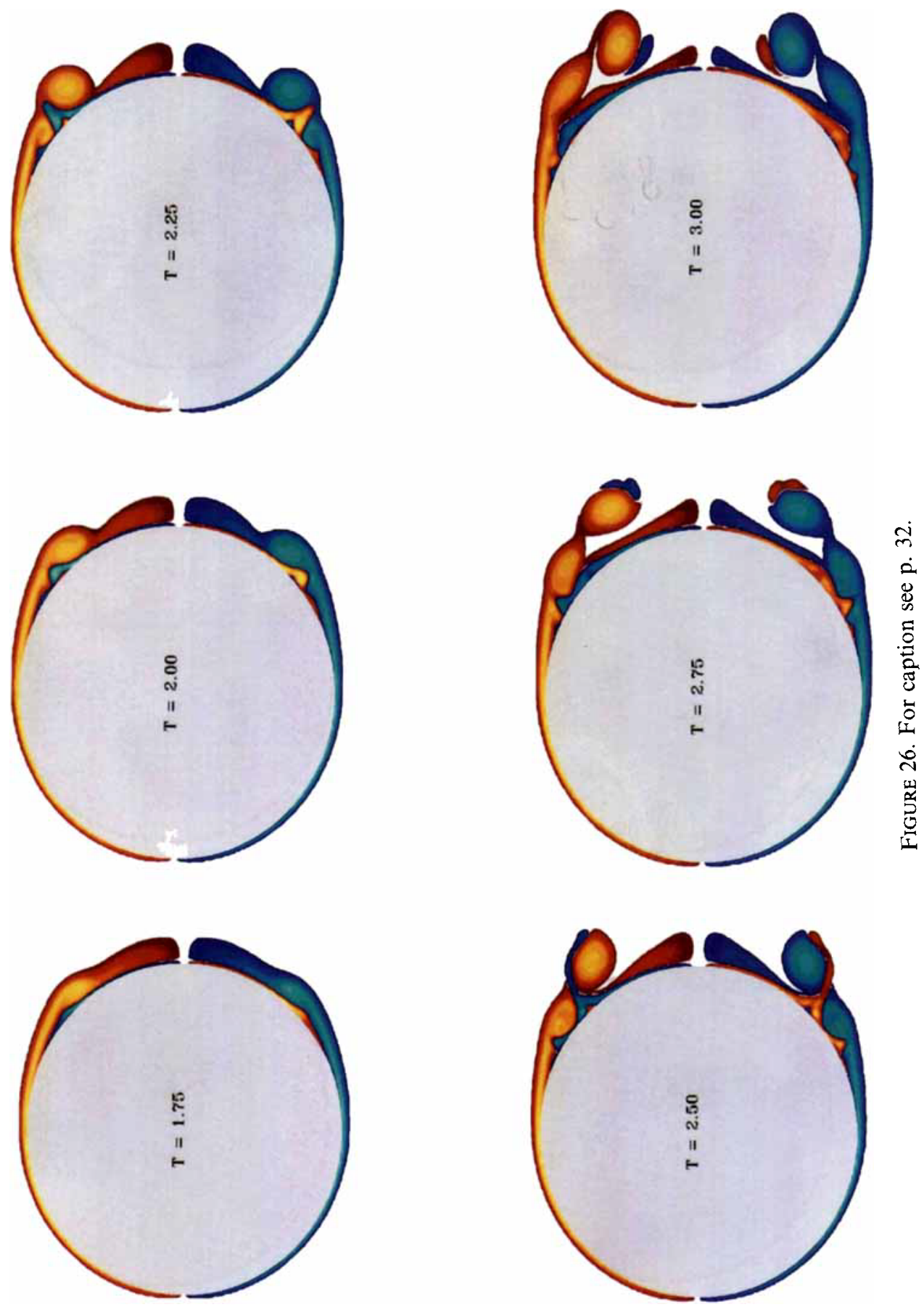

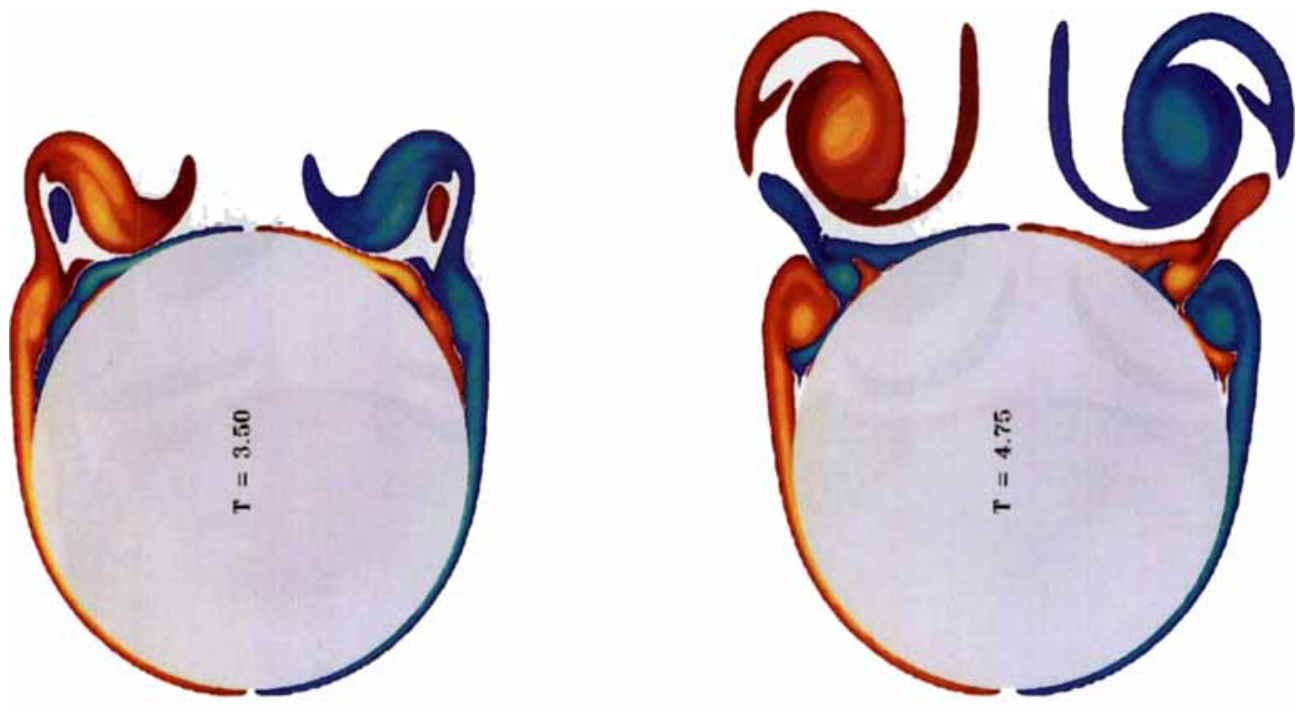

กं
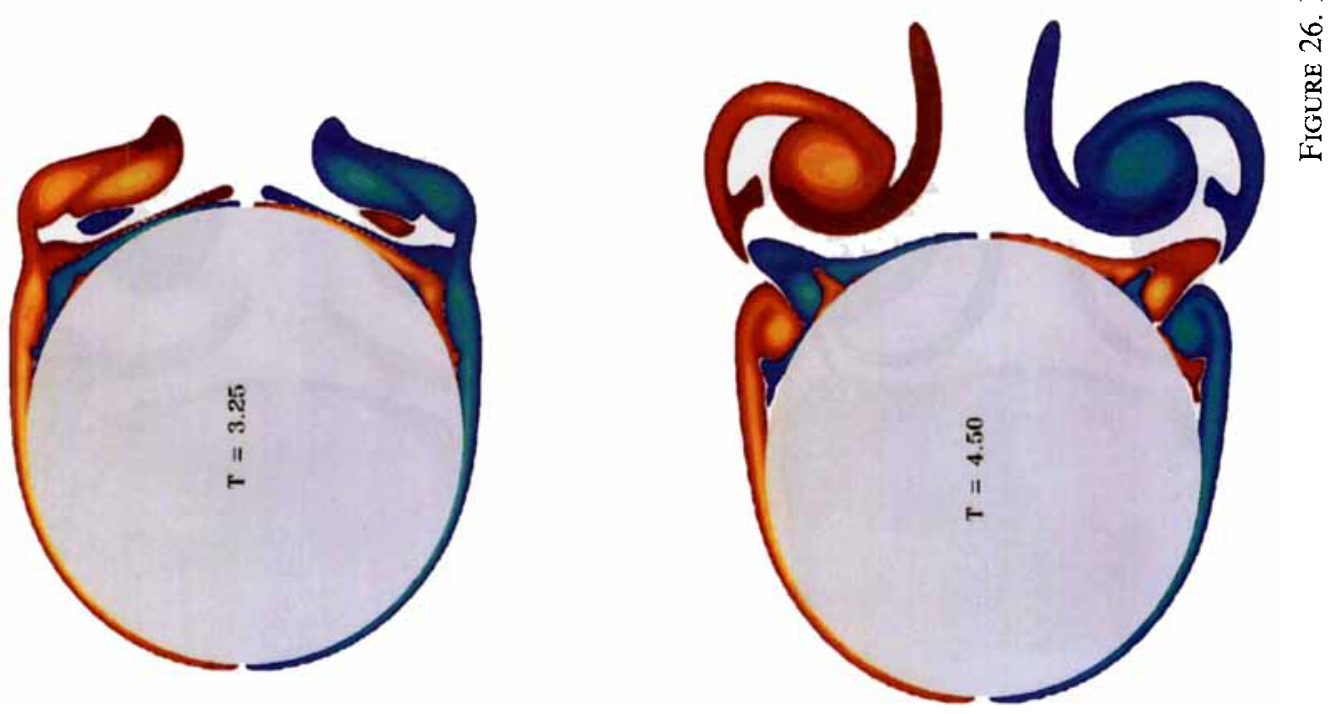

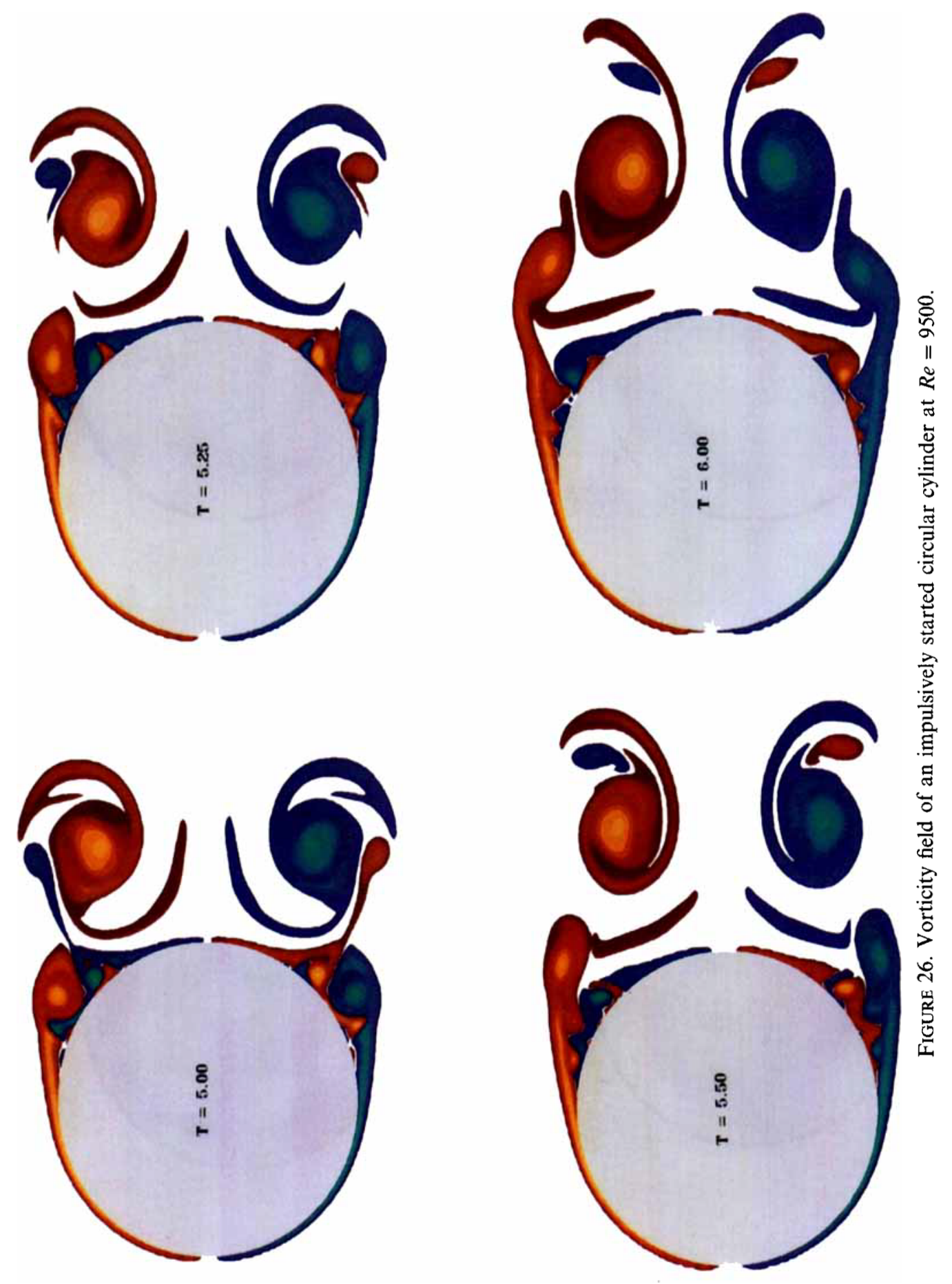


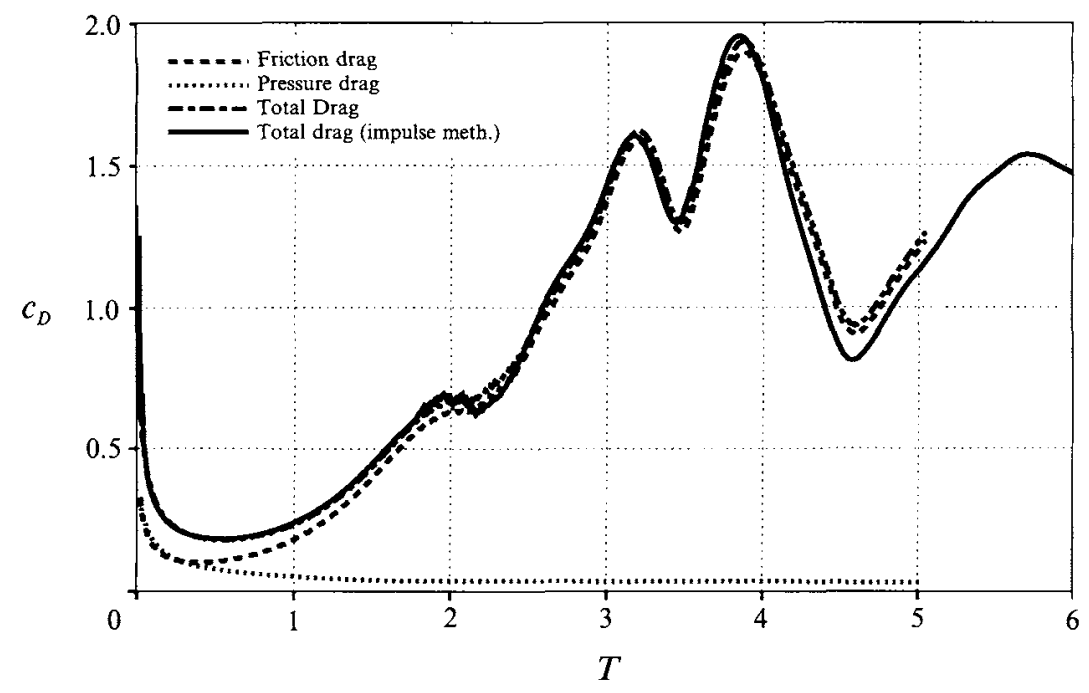

FIGURE 27. Evolution of the form and friction drag coefficient for $R e=9500$.

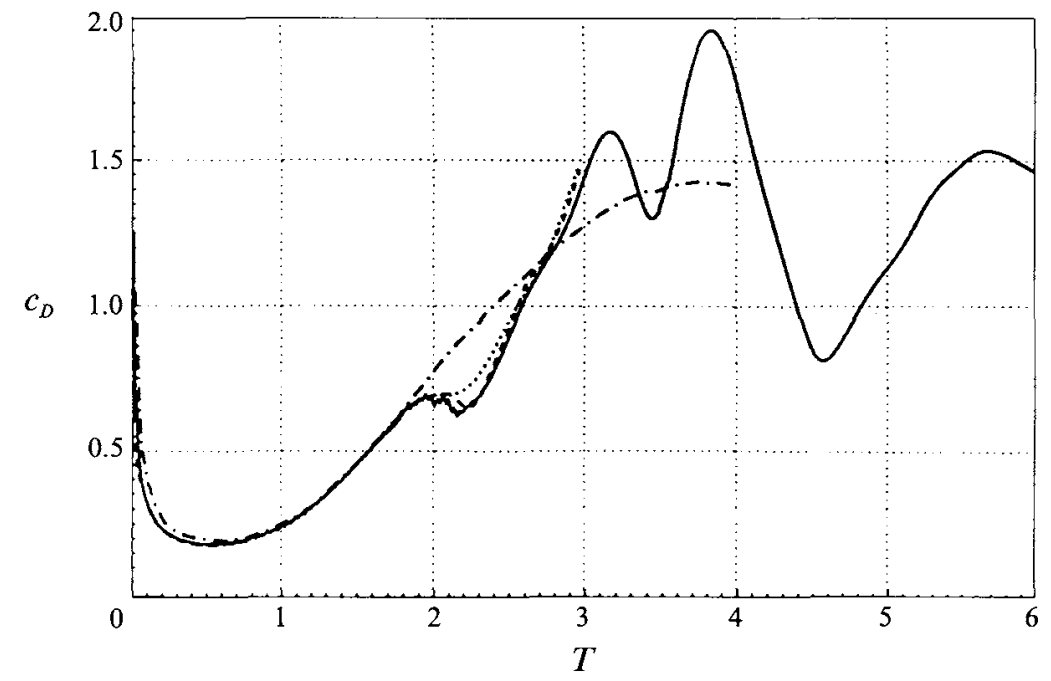

FIGURE 28. Comparison of the drag coefficient of an impulsively started circular cylinder at $R e=$ 9500 , as computed by various numerical schemes: - , present; ----, Anderson \& Reider; $\cdots$, Wu et al.; $-. \cdot-\cdot, \mathrm{CC}$.

of the cylinder for the various $R e$ : the evolution of the production of circulation follows the trends of the drag coefficient. The values presented herein may be implemented in simplified models of unsteady separated flows where an estimate for the production of circulation is necessary.

\section{Conclusions}

The present simulations present a Lagrangian-type numerical scheme, capable of resolving unsteady separated flows over a wide range of Reynolds numbers. Our 

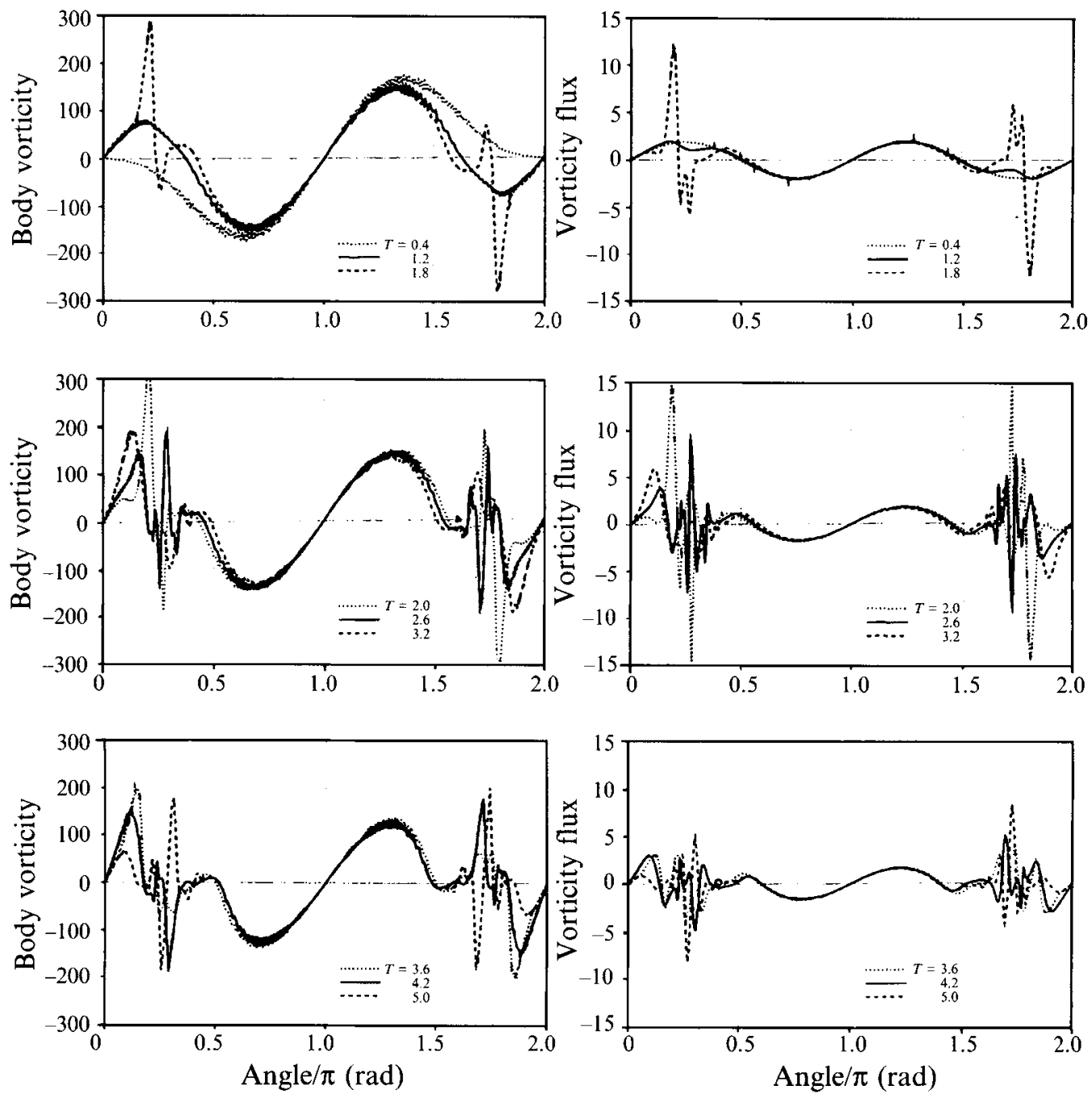

FIGURE 29. Vorticity on the surface of the body and vorticity flux $(\nu \partial \omega / \partial r)$ for $R e=9500$.

numerical method is capable of handling the abrupt eruptions of the boundary layers, associated with unsteady flows at high $R e$, as computational elements automatically adjust to resolve such regions. We gain insight in the interactions between vortical structures and walls and their effect on the forces experienced by the body, and our results elucidate the mechanisms of unsteady separation, especially at higher $R e$.

Through the present simulations we have identified two mechanisms by which nearwake vorticity directly affects the drag coefficient. The separating shear layer is the primary source of vorticity shed in the wake. However, when such shedding occurs in the vicinity of the boundary it induces a secondary flow and the generation of secondary vorticity. The interplay of this primary and secondary vorticity is the underlying mechanism for drag reduction and increase. The secondary vorticity once generated is further augmented by the presence of the primary vortex and eventually penetrates the link between the wake vortices and the shear layer and spills out to the outer flow. However, this excursion seems to be quite fatal for it as it is in turn 

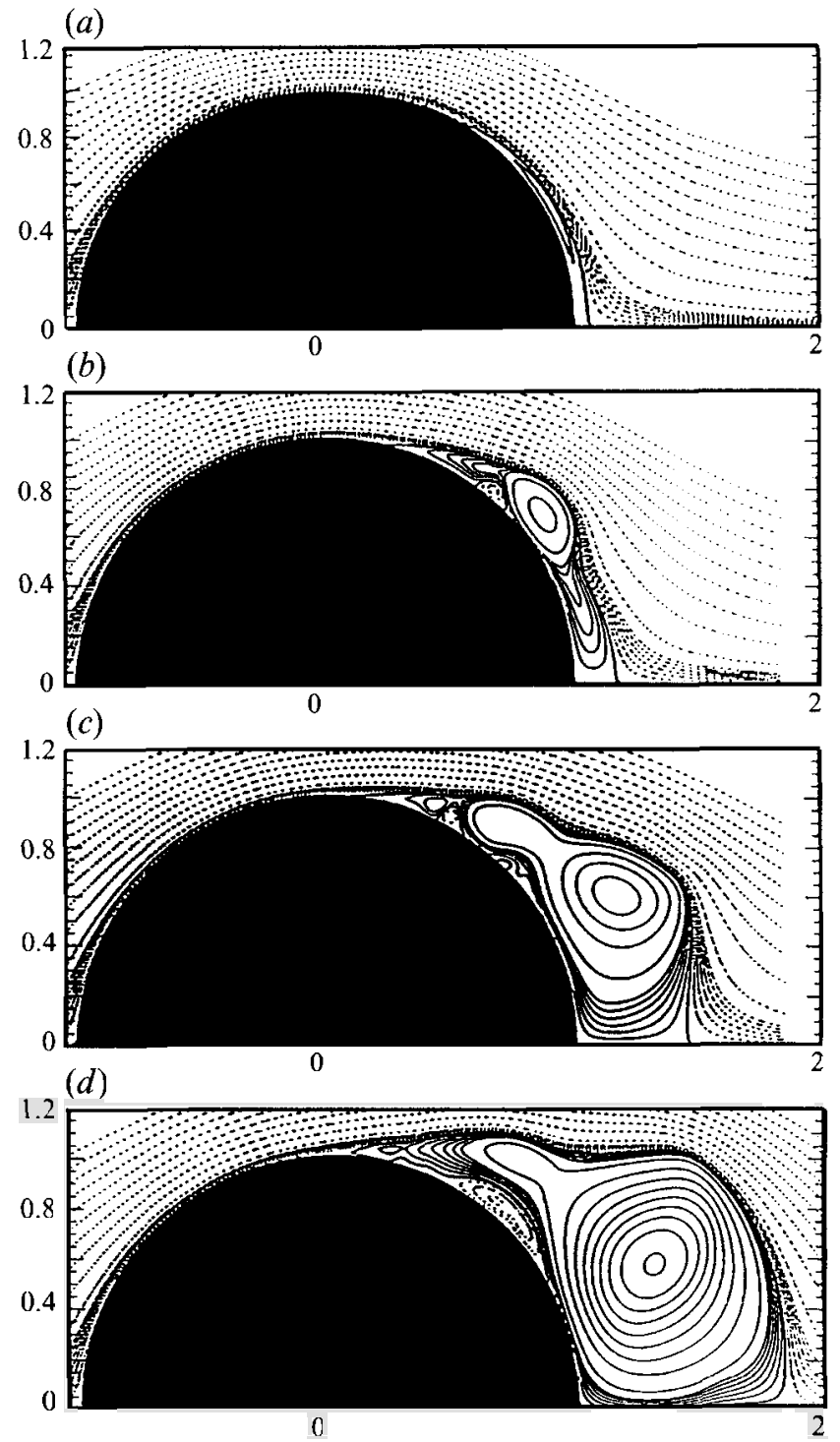

FIGURE 30. Instantaneous streamlines for an impulsively started circular cylinder at $R e=9500 ;(a) T=1,(b) T=2,(c) T=3,(d) T=4$.

elongated and severed by the primary vortex. As it now has no means of being selfsustained it is again over-run by the ever-present shear layers, thus starting a new cycle of events. This mechanism may be exploited by wake control devices (such as base suction and bleeding) that would affect the interplay of primary and secondary vorticity. For example blowing at a time and location where the secondary vorticity is confined by the primary shear layer would allow the penetration of the secondary vorticity to the outer flow, thus reducing the drag coefficient.

Our present high-resolution simulations have enabled advances in the understanding of unsteady separation at high Reynolds number, while providing benchmark quality simulations for the early stages of the flow development of an impulsively started circular cylinder. 

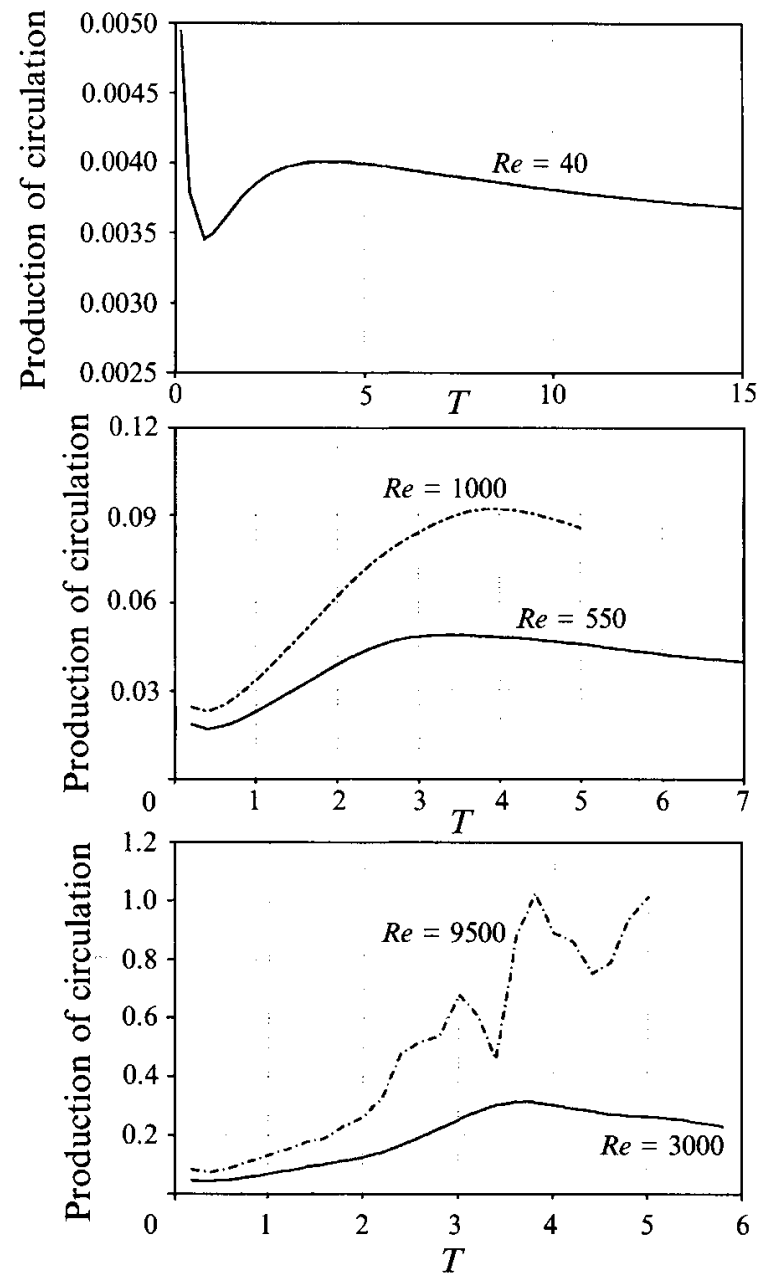

FIGURE 31. Production of circulation from the upper part of the impulsively started cylinder for $R e=40$ to 9500 as predicted by the present computations.

We would like to acknowledge the invaluable help of Mr Doug Shiels of Caltech throughout this work. We are pleased to acknowledge the support of this research by the Office of Naval Research through Grant Nos. N00014-92-J-1072 and N00014-92J-1189. One of us (P.K.) was an NSF postdoctoral fellow in parallel computing (Grant No. ASC-92-11071). Computer time was provided by Caltech's Jet Propulsion Laboratory Supercomputing Project.

\section{REFERENCES}

ANDERson, C. R. \& Reider, M. 1993 Investigation of the use of Prandtl/Navier--Stokes equation procedures for two-dimensional incompressible flows. CAM Rep. 93-02. Department of Mathematics, UCLA.

BAR-LeV, M. \& YANG, H. T. 1975 Initial flow field over an impulsively started circular cylinder. $J$. Fluid Mech. 72, 625-647 (referred to herein as BY).

BARnEs, J. E. \& Hut, P. 1986 A hierarchical $O(N \log N)$ force-calculation algorithm. Nature 324, 446-449. 
BEAle, J. T. 1986 On the accuracy of vortex methods at large times. Proc. Workshop on Comp. Fluid Dyn. and React. Gas Flows, IMA, University of Minnesota.

Blasıus, H. 1908 Grenzchichten in Flüssigkeiten mit Kleiner Reibug. Z. Angew. Math. Phys. (Engl. transl.) $N A C A T M-125656$, p. 1.

Bouard, R. \& Coutanceau, M. 1980 The early stage of development of the wake behind an impulsively started cylinder for $40 \leqslant R e \leqslant 10^{4}$. J. Fluid Mech. 101, 583-607 (referred to herein as $\mathrm{BC}$ ).

BrySON, A. E. 1959 Symmetric vortex separation on circular cylinders and cones. Trans. ASME E: J. Appl. Mech. 11, 643.

Chang, C. C. \& Chern, R. L. 1991 A numerical study of flow around an impulsively started circular cylinder by a deterministic vortex method. J. Fluid Mech. 233, 243-263 (referred to herein as CC).

Christiansen, J. P. 1973 Vortex methods for flow simulation. J. Comput. Phys. 13, 363.

Collins, W. M. \& Dennis, S. C. R. $1973 a$ The initial flow past an impulsively started circular cylinder. Q. J. Mech. Appl. Maths 26, 53-75 (referred to herein as CD).

Collins, W. M. \& Dennis, S. C. R. $1973 b$ Flow past an impulsively started circular cylinder. $J$. Fluid Mech. 60, 105-127 (referred to herein as CD).

Degond, P. \& Mas-Gallic, S. 1989 The weighted particle method for convection-diffusion equations, Part I: The case of an isotropic viscosity, Part II: The anisotropic case. Math. Comput. 53, 485-526.

Dennis, S. C. R. \& KocabiYIK, S. 1991 An asymptotic matching condition for unsteady boundarylayer flows governed by the Navier-Stokes equations. IMA J. Appl. Maths 47, 81-98.

Doligaski, T. L., Smith, C. R. \& WalkeR, J. D. A. 1994 Vortex interactions with walls. Ann. Rev. Fluid Mech. 26, 573-616.

Friedman, A. 1966 Partial Differential Equations of Parabolic Type. Prentice-Hall.

Greengard, L. \& Rokhlin, V. 1987 A fast algorithm for particle simulations. J. Comput. Phys. 73, $325-348$.

Koumoutsakos, P. 1993 Direct numerical simulations of unsteady separated flows using vortex methods. PhD thesis, California Institute of Technology.

KOUMOUTSAKOS, P. \& LEONARD, A. 1993 Improved boundary integral method for inviscid boundary condition applications. AIAA J. 31, 401-404.

Koumoutsakos, P., Leonard, A. \& PePin, F. 1994 Boundary conditions for viscous vortex methods. J. Comput. Phys. 113, 52.

LeCOINTE, Y. \& Piquet, J. 1984 On the use of several compact methods for the study of the incompressible viscous flow around a circular cylinder. Computers Fluids 12, 255-280.

LighthiLl, M. J. 1963 Introduction. In Boundary Layer Theory (ed. J. Rosenhead), pp. 54-61. Oxford University Press.

PAYNe, R. B. 1958 Calculation of viscous unsteady flows past a circular cylinder. J. Fluid Mech. 4, $81-86$.

Prager, W. 1928 Die Druckverteilung an Körpern in ebener Potentialströmung. Physik. Z. 29, 865-869.

Prandtl, W. 1925 The magnus effect and windpowered ships. Wissenschaften 13, 93-108.

Smith, P. A. \& Stansby, P. K. 1988 Impulsively started flow around a circular cylinder by the vortex method. J. Fluid Mech. 194, 45-77 (referred to herein as SS).

TA PHuOC LoC 1980 Numerical analysis of unsteady secondary vortices generated by an impulsively started circular cylinder. J. Fluid Mech. 100, 111-128 (referred to herein as TL).

TA Phuoc Loc \& Bouard, R. 1985 Numerical solution of the early stage of the unsteady viscous flow around a circular cylinder: a comparison with experimental visualization and measurements. J. Fluid Mech. 160, 93-117.

TRITton, D. J. 1959 Experiments on the flow past a circular cylinder at low Reynolds numbers. $J$. Fluid Mech. 6, 547-567.

Van Dommelen, L. L. \& Shen, S. F. 1980 The spontaneous generation of the singularity in a separating boundary layer. J. Comput. Phys. 38, 125-140. 
WANG, X. \& Dalton, C. 1991 Numerical solutions for impulsively started and decelerated viscous flow past a circular cylinder. Intl J. Numer. Meth. Fluids 12, 383-400.

WU, J. C. 1976 Numerical boundary conditions for viscous flow problems. AIAA J. 14, 1042-1049.

WU, J.-Z., WU, X., MA, H. \& WU, J.-M. 1995 Dynamic vorticity condition: theory and numerical implementation. Intl J. Numer. Meth. Fluids (submitted). 